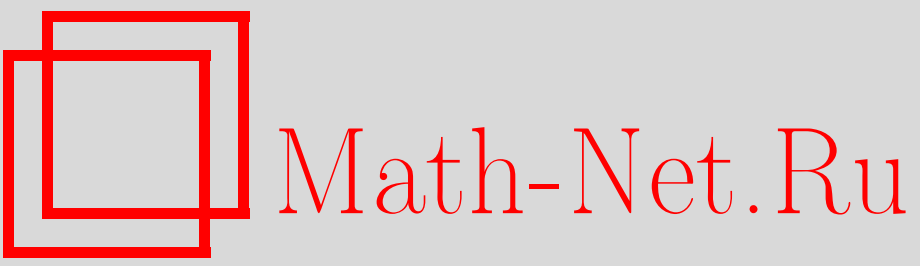

С. А. Богатый, Геометрия отображений в евклидово пространство, УМН, 1998, том 53, выпуск 5, 27-56

DOI: https://doi.org/10.4213/rm68

Использование Общероссийского математического портала Math-Net.Ru подразумевает, что вы прочитали и согласны с пользовательским соглашением

http://www.mathnet.ru/rus/agreement

Параметры загрузки:

IP : 18.234 .156 .22

26 апреля 2023 г., 03:10:49 


\title{
ГЕОМЕТРИЯ ОТОБРАЖЕНИЙ В ЕВКЛИДОВО ПРОСТРАНСТВО
}

\author{
С. А. БОГАТЫЙ
}

\section{СОДЕРЖАНИЕ}

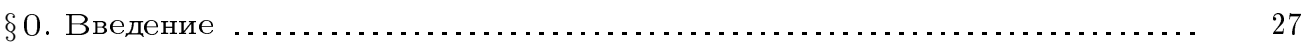

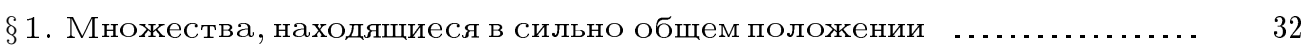

$\S 2$. Регулярно ветвящиеся отображения .......................... 37

$\S 3$. Обращение теоремы Гуревича для полиэдров $\ldots \ldots \ldots \ldots \ldots \ldots \ldots \ldots \ldots . . \ldots \ldots \ldots$

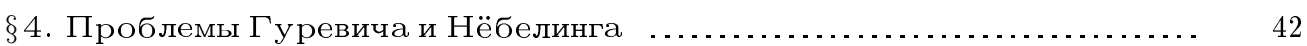

$\S 5$. Обращение теоремы существования для полиэдров . ............... 44

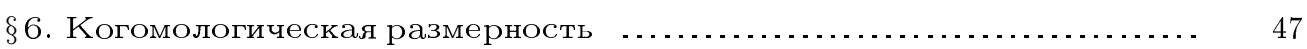

$\S 7$. Исторические замечания к формуле Урысона .................... 50

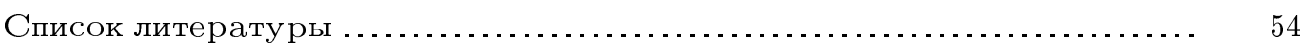

\section{$\S$ 0. Введение}

В разных областях математики имя П. С. Урысона ассоциируется с разными понятиями и результатами [1]. В нелинейном анализе это, несомненно, в первую очередь, интегральньй оператор Урысона $[2$, гл. $1, \S 3]$, в выпуклой геометрии это неравенство Урысона [3, с. 65], в теории аппроксимаций это поперечник Урысона [4, с. 18], а в топологии это, конечно, теория бикомпактных пространств и теория размерности (пространство Фреше-Урысона, теорема Брауэра-Урысона-Титце о продолжении, формула Урысона).

Напомним мнение П. С. Александрова [5, с. 10], [1, т. 1, с. 33] о том, что только теорема Урысона о совпадении индуктивной размерности и лебеговой размерности компакта позволила связать интуитивно понятное определение размерности с определением, которое явилось основой всего дальнейшего развития теории размерности. Здесь уместно подчеркнуть, что хотя определение размерности с помошью кратности мелких покрытий и восходит к работам Лебега [6], [7] и Брауэра [8], но именно в доказательстве своего тождества $\operatorname{dim} X=\operatorname{ind} X=\operatorname{Ind} X$ П. С. Урысон выделил $\operatorname{dim} X$ в качестве особого топологического инварианта компакта $X$, а не остался в пределах

Работа выполнена при поддержке Российского фонда фундаментальных исследований (грант № 97-01-00357) и INTAS (грант № 96-0712). 
работ Лебега и Брауэра о кратности покрытий $n$-мерного куба. Бегльй взгляд на содержание монографий по теории размерности, на программы конференций по общей, геометрической и алгебраической топологии показьвает, что именно инвариант $\operatorname{dim}$ ( "краеугольньй камень теории размерности” по мнению Р. Энгелькинга и Р. Поля) явился основой всего последуюшего развития теории размерности. "Одна из самых больших заслуг Урысона в теории размерности состоит в том, что он впервые выделил (1921 г.) размерность $\operatorname{dim} X$ в качестве самостоятельного топологического инварианта и доказал (для компактов $X$ ) тождество $\operatorname{dim} X=\operatorname{ind} X=\operatorname{Ind} X$ " [9, с. 164, сноска $\left.\left.{ }^{*}\right)\right]$. Именно важность размерности $\operatorname{dim}$ заставляет Р. Энгелькинга и Р. Поля отмечать в специальной сноске (в работе, посвященной В. Гуревичу [10]), что размерность $\operatorname{dim}$ для нормальных пространств была введена Э. Чехом (см. $\S 7$ ).

“Эта эквивалентность двух совершенно разнородных определений позволяет, естественно, взглянуть на урысоновскую размерность с совершенно новой комбинаторно-алгебраической точки зрения. Таким образом, открьвается широкая возможность приложения алгебраических методов к изучению размерности и связанных с нею топологических свойств" [1, т. 1 , с. 37$]$.

Именно комбинаторно-топологическая теорема П. С. Александрова, характеризующая размерность dim с помошью $\varepsilon$-отображений в полиэдры, позволяет легко получить оценку сверху размерности произведения. Для любых компактных метрических пространств $X$ и $Y$ справедливо неравенство

$$
\operatorname{dim} X \times Y \leqslant \operatorname{dim} X+\operatorname{dim} Y .
$$

Попытки получения в логарифмическом неравенстве (log) равенства явились источником развития многих областей алгебраической топологии. Л.С. Понтрягин [11] построил примеры двумерных компактов, произведение которых трехмерно. В.Г. Болтянский [12] построил пример двумерного компакта, квадрат которого трехмерен. М.Ф. Бокштейн [13] полностью решил задачу вычисления размерности произведения двух компактов. Когда содержание данной работы рассказьвалось в июне 1997 года на семинаре Х. Цишанга в Ruhr-Universität Bochum, то некоторые участники семинара, интересы которых находятся, в основном, в области алгебры и маломерной топологии, недоумевали по поводу “неправильности” размерности (или класса рассматриваемых пространств), которая при взятии произведения не складывается. Более чем 70-летняя история развития общей топологии после публикации мемуаров П.С. Александрова и П. С. Урысона по теории бикомпактных пространств и теории размерности показьвает как естественность в общей топологии класса метрических бикомпактных (компактных) пространств, так и “единственность" (а значит, “правильность") размерности. Конечно, в алгебраической топологии более естественным является класс $C W$-комплексов, а в гладкой топологии - класс многообразий.

Можно сказать, что эта "единственная неправильность" поведения размерности оказала самое сильное влияние на развитие и даже возникновение других областей математики. Так, по мнению Е.Г. Скляренко, именно в работах М. Ф. Бокштейна по вычислению размерности произведения возникли основы теории когомологических операций и гомологической алгебры, что, к сожалению, не нашло должного отражения в соответствующей исторической литературе. 
Хотя основным вкладом П. С. Урысона в математику, видимо, считается построение теории размерности, но, скорее всего, наибольшую известность П.С. Урысон имеет в связи с понятием "поперечник Урысона". Приведем выдержку из монографии К.И. Бабенко [14]. “Замечательно, что поперечники впервые появились в работе П. С. Урысона, написанной в 1925, 1926 гг. [15] и посвященной теории размерности. Важные в численном анализе поперечники Александрова введены в 1933 г. в работе [16], но опять не в связи с теорией приближений."

В настоящее время вычисление различных поперечников (например, Александрова, Бернштейна, Гельфанда, Колмогорова, Урысона [4, с. 14-18]) различных (функциональных) пространств является одной из основных задач теории аппроксимаций. Поперечник Урысона является развитием понятия размерности dim.

Процитируем еще раз П. С. Александрова [1, т. 1, с. 41]. "Урысону принадлежит общий почин рассмотрения конечных замкнутых покрытий, сделавшихся впоследствии одним из основных инструментов топологии, в частности, явившихся средством построения гомологической теории компактов и значительно более общих пространств. С другой стороны, привлечение тех же покрытий послужило основой для введения Л.А. Люстерником и Л.Г. Шнирельманом понятия категории, позволившего им, как известно, далеко развить топологические методы в вариационном исчислении и решить при их помоши ряд классических задач."

В недавней работе автора [17] рассматривается некоторый аналог теоремы Люстерника-Шнирельмана для действия произвольной конечной групшы. Данная статья посвящена аналогу поперечника Александрова, в котором, правда, рассматриваются не две склеивающиеся точки, а $k$ точек, имеюших обший образ, но отображения берутся не в произвольный полиэдр, а в евклидово пространство (многоточечный аналог поперечника $\gamma_{m}(X)[4$, с. 16]). Так как в работе исследуется только факт существования соответствующих склеивающихся $k$ точек, а не их метрический инвариант, т.е. исследуется только вопрос зануления или не зануления соответствующего аналога поперечника Александрова, то фактически все понятия теории поперечников остаются за кадром. Сам автор пришел к рассматриваемым в статье задачам, изучая теоремы типа Борсука-Улама, Люстерника-Шнирельмана о склеивании некоторой орбиты действия группы $\mathbb{Z}_{p}$ или, наоборот, отсутствия такого склеивания, полученного В. В. Макеевым при опровержении гипотезы Кнастера с помошью рассмотрения конфигураций и отображений общего положения [18]-[21]. Только впоследствии автор узнал, что метод общего положения много ранее успешно применялся Гуревичем [22]-[23] для построения отображений малой кратности (т.е. для "расцепления" всех $k$-точечных конфигураций). Так как на $k$-й степени любого пространства действие группы $\mathbb{Z}_{k}$ задается совершенно естественным образом, то это действие, опять-таки, очень часто остается за кадром. Но так как индекс свободного действия групшы $\mathbb{Z}_{p}$ на пространстве $X^{p} \backslash \Delta$ оценивается сверху размерностью пространства $X^{p}$, то мы видим важность вычисления в таких задачах размерности если и не общего произведения компактов, то, по крайней мере, степеней компактов.

Классическая теорема Нёбелинга-Понтрягина-Лефшеца (доказанная в одномерном случае также Менгером) о вложении утверждает, что всякий компакт $X$ размерности $\operatorname{dim} X=n$ вкладьвается в $(2 n+1)$-мерное евклидово пространство $\mathbb{R}^{2 n+1}$. Обьчно в монографиях по теории размерности теорема вложения дается в более сильной формулировке В. Гуревича [9], [24]. Множество всех вложений $n$-мерного 
компактного пространства $X$ в т-мерное евклидово пространство $\mathbb{R}^{m}$ при $m \geqslant 2 n+1$ всюду плотно в пространстве $\mathscr{C}\left(X, \mathbb{R}^{m}\right)$ всех непрерывных отображений компакта $X$ в $\mathbb{R}^{m}$.

В действительности В. Гуревич доказал значительно более сильное утверждение [22]. Мы будем следовать терминологии работы [25]. Для отображения $f: X \rightarrow Y$ и всякого $k \geqslant 0$ рассмотрим подмножество $B_{k}(f) \subseteq Y$ :

$$
B_{k}(f)=\left\{y \in Y: \operatorname{card} f^{-1}(y) \geqslant k\right\} .
$$

Отображение $f$ называется регулярно ветвящимся, если для всякого $k \geqslant 1$ имеет место неравенство

$\left(H_{k}\right) \quad \operatorname{dim} B_{k}(f) \leqslant k \operatorname{dim} X-(k-1) \operatorname{dim} Y=\operatorname{dim} Y-k(\operatorname{dim} Y-\operatorname{dim} X)$.

Очевидно, что при $2 \operatorname{dim} X+1 \leqslant \operatorname{dim} Y$ всякое регулярно ветвяшееся отображение является вложением. Кроме того, если $\operatorname{dim} X+1 \leqslant \operatorname{dim} Y$, то всякое регулярно ветвящееся отображение имеет кратность $\leqslant \operatorname{dim} X+1$. Для регулярно ветвяшегося отображения $f$ справедливо также неравенство $\operatorname{dim} f(X) \leqslant \operatorname{dim} X$.

ТеОрема ГУРеВИЧА. Если $m \geqslant \operatorname{dim} X+1$, то множество всех регулярно ветвящихся отображсений $\mathscr{H}\left(X, \mathbb{R}^{m}\right)$ компакта $X$ в $\mathbb{R}^{m}$ содерэсит всюду плотное в пространстве $\mathscr{C}\left(X, \mathbb{R}^{m}\right)$ множсество типа $G_{\delta}$.

Мы показьваем, что при $m \geqslant \operatorname{dim} X+3$ из теоремы Дранишникова [26] вытекает возможность замены в теореме Гуревича в условии $\left(H_{2}\right)$ числа $2 \operatorname{dim} X$ на не большее число $\operatorname{dim} X^{2}$. При $m=\operatorname{dim} X+1$ такая замена невозможна.

Для любых целых чисел $r \geqslant-1$ и $k \geqslant 1$ рассмотрим в $\mathscr{C}(X, Y)$ подмножество $\mathscr{N}_{r, k}(X, Y)$ всех таких отображений, для которых имеет место неравенство $\operatorname{dim} B_{k}(f) \leqslant r$.

Понятно, что при $k \operatorname{dim} X-(k-1) \operatorname{dim} Y \leqslant r$ имеет место включение

$$
\mathscr{H}(X, Y)=\bigcap_{k} \mathscr{N}_{k \operatorname{dim} X-(k-1) \operatorname{dim} Y, k}(X, Y) \subseteq \mathscr{N}_{r, k}(X, Y)
$$

Теорему Гуревича можно ослабить до следующего утверждения.

Если числа $n=\operatorname{dim} X, m, k$ и $r$ удовлетворяют неравенству

$$
k n \leqslant(k-1) m+r,
$$

то мнохсество $\mathscr{N}_{r, k}\left(X, \mathbb{R}^{m}\right)$ всюду плотно в пространстве $\mathscr{C}\left(X, \mathbb{R}^{m}\right)$.

СЛЕДСТВИЕ ГУРЕВИЧА. Если числа $n, m$ u $k$ удовлетворяют неравенству

$(n, m, k) \quad n+1 \leqslant(m-n) k$,

то для любого п-мерного компакта $X$ множество всех его не более чем $k$-кратных отображсний в плотное в пространстве $\mathscr{C}\left(X, \mathbb{R}^{m}\right)$ подмножество типа $G_{\delta}$.

В. Гуревичем была сформулирована следующая гипотеза обрашения [22, с. 400]. 
"Мне кажется очень вероятным, что $n$-мерные компакты характеризуются тем, что множество их вложений в $\mathbb{R}^{2 n+1}$ плотно в пространстве $\mathscr{C}\left(X, \mathbb{R}^{2 n+1}\right) . "$

Нёбелинг сформулировал более общую гипотезу [27].

"Вероятно для компакта $X$ неравенство $\operatorname{dim} X \leqslant n$ равносильно (при заданном числе $r,-1 \leqslant r \leqslant n-1)$ плотности в пространстве $\mathscr{C}\left(X, \mathbb{R}^{2 n-r}\right)$ подмножества всех конечнократных отображений из $\mathscr{N}_{r, 2}\left(X, \mathbb{R}^{2 n-r}\right) . "$

Теорема Бокштейна-Кузьминова [28] показьвает, что положительньй ответ на проблему Гуревича содержится в теореме Дранишникова-Веста [29]. Здесь уместно отметить, что, тесно связанная с указанной проблемой Гуревича, проблема описания $n$-мерных компактов $X$, множество вложений которых в $\mathbb{R}^{2 n}$ всюду плотно, полностью решена [30].

Положительное же решение проблемы Нёбелинга вытекает из теоремы Бокштейна-Кузьминова и теоремы Дранишникова-Реповша-Шепина [31] .

В случае полиэдра $X$ мы доказываем обрашение ослабленного варианта теоремы Гуревича при произвольных числах $r$ и $k$. Обращение ослабленного варианта теоремы Гуревича для полиэдральных пространств получается в работе с помощью понятия подмножества $\mathbb{R}^{m}$, находящегося в сильно общем положении. Рассмотрение подмножеств $\mathbb{R}^{m}$, находяшихся в сильно обшем положении, позволило дать простое доказательство теоремы о размерности пересечения образов обших отображений и получить некоторое усиление теоремы Тверберг [32].

В пятом параграфе работы получены некоторые результаты об обрашении теоремы существования "экономичного” отображения (при отсутствии требования плотности множества “экономичных" отображений). В случае, когда число $k+1$ является двойкой, простьм нечетным числом, степенью простого числа, обрашение следствия Гуревича о существовании не более чем $k$-кратного отображения дается теоремами ван Кампена и Флореса [33], [34], Саркариа [35], Воловикова [36]. С помощью теоремы Коэна-Ласка [37] о частичной склейке орбиты мы получаем некоторое слабое обращение следствия Гуревича о существовании не более чем $k$-кратного отображения в случае произвольного числа $k+1$, которое, однако, при $m=n+1$ позволяет получить полное обращение указанного следствия Гуревича. Именно, $n p u \quad N=2 n^{2}+5 n$ для всякого непрерывного отображения $f: \Delta_{n}^{N} \rightarrow \mathbb{R}^{n+1}$, где $\Delta_{n}^{N}$ обозначает $n$-мерный остов $N$-мерного симплекса, существуют такие попарно дизбюнктные замкнутые симплексы $\sigma_{1}, \ldots, \sigma_{n+1} \subset \Delta_{n}^{N}$, что пересечение их образов непусто. Автору неизвестно, можно ли в сформулированном утверэдении взять $N=n^{2}+3 n$. Компакт $\Delta_{n}^{N}$ является полиэдром с “достаточным" количеством $n$-мерных симплексов, которые сильно сцеплены. Но оказалось, что при $m=n+1$ есть еще один класс пространств, у которых любое отображение сильно отличается от вложения.

Именно, мы доказьваем следуюшее усиление теоремы Александрова о размерной полноценности $n$-мерного компакта в $\mathbb{R}^{n+1}$. У всякого нульмерного отображсения $f$ $n$-мерного размерно неполноченного компакта в $\mathbb{R}^{n+1}$ множество точек неоднократности $\left(\right.$ в $\left.\mathbb{R}^{n+1}\right)$ имеет размерность не менее $n-1$, m.е. $\operatorname{dim} B_{2}(f) \geqslant n-1$. Сформулированное усиление теоремы Александрова получено в качестве следствия некоторого когомологического варианта теоремы Фрейденталя-Вайнштейна, которая соответствует когомологическому варианту Кузьминова теоремы Гуревича о повышающих размерность конечнократных отображениях.

Мы также показываем, что для конечномерных компактов алгебраический и 
геометрический дефекты равны, что является усилением характеризации Болтянского размерно полноценных компактов [38]. Наше доказательство опирается на вычисления в алгебре Бокштейна, проведенные для других целей А. Н. Дранишниковым, Д. Реповшем и Е. В. Шепиным [39].

В седьмом параграфе обсуждается историческая проблема о возникновении общеупотребительного ньне термина “формула Урысона-Менгера”, ответ на которую, видимо, никогда уже не будет получен.

\section{§ 1. Множества, находящиеся в сильно общем положении}

Для множества $M \subseteq \mathbb{R}^{m}$ пусть $\Pi(M)$ означает наименьшую плоскость в $\mathbb{R}^{m}$, содержащую это множество $M$. Плоскость П $(M)$ существует и единственна и

$$
\Pi(M)=\bigcap\left\{\Pi: \Pi \supseteq M \text { и П это плоскость в } \mathbb{R}^{m}\right\} \text {. }
$$

Для упрощения обозначений (и по аналогии с подпространствами в векторном пространстве) наименьшую плоскость, содержащую заданные плоскости $\Pi_{1}$ и $\Pi_{2}$, т.е. плоскость $\Pi\left(\Pi_{1} \cup \Pi_{2}\right)$, будем обозначать через $\Pi_{1}+\Pi_{2}$.

ПРЕДЛОЖЕНИЕ 1. Если плоскости $\Pi_{1}$ и $\Pi_{2}$ пересекаются, то справедливо равенство

$$
\operatorname{dim} \Pi_{1} \cap \Pi_{2}=\operatorname{dim} \Pi_{1}+\operatorname{dim} \Pi_{2}-\operatorname{dim}\left(\Pi_{1}+\Pi_{2}\right) .
$$

ПРЕДЛОЖЕНИЕ 2. Если плоскости $\Pi_{1}, \ldots, \Pi_{k}$ пересекаются, то справедливо неравенство

$$
\operatorname{dim} \Pi_{1} \cap \cdots \cap \Pi_{k} \geqslant \operatorname{dim} \Pi_{1}+\cdots+\operatorname{dim} \Pi_{k}-m(k-1) .
$$

ОПРЕДЕЛЕНИЕ 1 . Множество точек $M$ пространства $\mathbb{R}^{m}$ находится в общем положении, если для всякого подмножества $M_{1} \subseteq M$, содержащего не более чем $(m+1)$ точку, справедливо равенство $\operatorname{dim} \Pi\left(M_{1}\right)=\left|M_{1}\right|-1$.

ОПРЕДЕЛЕНИЕ 2. Множество точек $M$ пространства $\mathbb{R}^{m}$ находится в сильно общем положении, если любые попарно непересекающиеся подмножества $M_{1}, \ldots, M_{k} \subseteq M$ удовлетворяют условию

$(\varnothing)$ пересечение плоскостей $\Pi\left(M_{1}\right), \ldots, \Pi\left(M_{k}\right)$ пусто, или

$(\mathrm{SGP}) \quad(=) \quad \operatorname{dim} \Pi\left(M_{1}\right) \cap \cdots \cap \Pi\left(M_{k}\right)$

$$
=\operatorname{dim} \Pi\left(M_{1}\right)+\cdots+\operatorname{dim} \Pi\left(M_{k}\right)-m(k-1) .
$$

Так как условия $\Pi=\mathbb{R}^{m}$ и $\operatorname{dim} \Pi=m$ равносильны, то в определении 2 все подмножества $M_{i}$, для которых $\operatorname{dim} \Pi\left(M_{i}\right)=m$, можно не учитьвать. Такие подмножества не меняют ни правую, ни левую часть равенства $(=)$ в (SGP), следовательно без ограничения общности всегда можно считать, что $\operatorname{dim} \Pi\left(M_{i}\right) \leqslant m-1$. Далее, так как для оставшихся подмножеств справедливо неравенство $\operatorname{dim} \Pi\left(M_{i}\right) \leqslant m-1$, то для правой части равенства $(=)$ справедлива оценка

$$
\operatorname{dim} \Pi\left(M_{1}\right)+\cdots+\operatorname{dim} \Pi\left(M_{k}\right)-m(k-1) \leqslant(m-1) k-m(k-1)=m-k .
$$

Следовательно, при $m+1 \leqslant k$ в условиях определения 2 пересечение плоскостей всегда пусто, поэтому всегда можно считать, что $k \leqslant m+1$. Далее, так как в условии определения фигурирует только плоскость $\Pi\left(M_{i}\right)$, а не само первоначальное множество и взятие подмножеств в $M_{i}$ не уничтожает попарную дизъюнктность, то без ограничения общности всегда можно считать, что $\operatorname{dim} \Pi\left(M_{i}\right)=\left|M_{i}\right|-1$. 
ЗАмЕчАниЕ 1. В условии (=) из (SGP) вместо равенства можно поставить знак неравенства “ $\leqslant$ ". Действительно, согласно неравенству предложения 2 неравенство

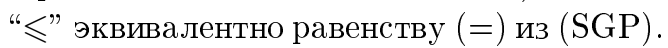

ЛЕмма 1. Для подмножсества точек $M \subset \mathbb{R}^{m}$ следующие условия әквивалентны.

(1) Множсество $M$ находится в общем положсении.

(2) Для любьх двух дизбюнктных непустых подмножеств $M_{1}, M_{2} \subset M$ та$\kappa и х$, что $\left|M_{1}\right|+\left|M_{2}\right| \leqslant m+1$, имеет место равенство $\Pi\left(M_{1}\right) \cap \Pi\left(M_{2}\right)=\varnothing$.

(3) Для любьх двух дизбюнктных непустых подмножсеств $M_{1}, M_{2} \subset M$ таких, что $\Pi\left(M_{1}\right) \cap \Pi\left(M_{2}\right) \neq \varnothing$, справедливо равенство $\operatorname{dim} \Pi\left(M_{1}\right) \cap \Pi\left(M_{2}\right)=$ $\operatorname{dim} \Pi\left(M_{1}\right)+\operatorname{dim} \Pi\left(M_{2}\right)-m$.

(4) Для любых двух непустых подмножеств $M_{1}, M_{2} \subseteq M$ справедливо по крайней мере одно из следующих равенств:

(a) $\Pi\left(M_{1}\right)+\Pi\left(M_{2}\right)=\mathbb{R}^{m}$;

(b) $\Pi\left(M_{1}\right) \cap \Pi\left(M_{2}\right)=\Pi\left(M_{1} \cap M_{2}\right)$.

ДокАЗАТЕльство. Последовательно докажем справедливость следуюших импликаций $(4) \Rightarrow(3) \Rightarrow(2) \Rightarrow(1) \Rightarrow(4)$.

$(4) \Rightarrow(3)$. Если $M_{1}, M_{2} \subset M$ это непустые дизъюнктные подмножества, то или имеет место равенство $\Pi\left(M_{1}\right) \cap \Pi\left(M_{2}\right)=\Pi\left(M_{1} \cap M_{2}\right)=\Pi(\varnothing)=\varnothing$, или $\Pi\left(M_{1}\right) \cap$ $\Pi\left(M_{2}\right) \neq \varnothing$, а тогда согласно предложению 1 и равенству (b) условия (4) справедливо равенство условия (3).

$(3) \Rightarrow(2)$. Возьмем такие непустые дизъюнктные подмножества $M_{1}, M_{2} \subseteq M$, что $\left|M_{1}\right|+\left|M_{2}\right| \leqslant m+1$. Если $\Pi\left(M_{1}\right) \cap \Pi\left(M_{2}\right) \neq \varnothing$, то согласно предложению 1 справедливо неравенство

$$
\begin{aligned}
\operatorname{dim} \Pi\left(M_{1}\right) \cap \Pi\left(M_{2}\right) & =\operatorname{dim} \Pi\left(M_{1}\right)+\operatorname{dim} \Pi\left(M_{2}\right)-m \\
& \leqslant\left(\left|M_{1}\right|-1\right)+\left(\left|M_{2}\right|-1\right)-m \\
& =\left|M_{1}\right|+\left|M_{2}\right|-2-m \leqslant m+1-2-m=-1,
\end{aligned}
$$

что противоречит центрированности рассматриваемых плоскостей.

$(2) \Rightarrow(1)$. Возьмем некоторое подмножество $M_{0} \subseteq M$, состоящее из не более чем $(m+1)$ точки. Если $\operatorname{dim} \Pi\left(M_{0}\right) \leqslant\left|M_{0}\right|-2$, то существует такая точка $A \in M_{0}$, что $A \in \Pi\left(M_{0} \backslash\{A\}\right)$. Тогда, положив $M_{1}=M_{0} \backslash\{A\}$ и $M_{2}=\{A\}$, мы получаем, что $\Pi\left(M_{1}\right) \cap \Pi\left(M_{2}\right)=A \neq \varnothing$.

$(1) \Rightarrow(4)$. Пусть $M_{1}, M_{2} \subseteq M$ и $\Pi\left(M_{1}\right)+\Pi\left(M_{2}\right) \neq \mathbb{R}^{m}$. Тогда согласно равенству $\Pi\left(M_{1}\right) \cup \Pi\left(M_{2}\right)=\Pi\left(M_{1}\right)+\Pi\left(M_{2}\right)$ получается, что из условия общего положения вытекает неравенство $\operatorname{dim} \Pi\left(M_{1} \cup M_{2}\right)=\left|M_{1} \cup M_{2}\right|-1 \leqslant m-1$. Теперь или плоскости П $\left(M_{1}\right)$ и П $\left(M_{2}\right)$ дизъюнктны, и тогда имеет место равенство (а) условия (4), или $\Pi\left(M_{1}\right) \cap \Pi\left(M_{2}\right) \neq \varnothing$ и тогда согласно предложению 1 справедливо равенство

$$
\begin{aligned}
\operatorname{dim} \Pi\left(M_{1}\right) \cap \Pi\left(M_{2}\right) & =\operatorname{dim} \Pi\left(M_{1}\right)+\operatorname{dim} \Pi\left(M_{2}\right)-\operatorname{dim} \Pi\left(M_{1} \cup M_{2}\right) \\
& =\left|M_{1}\right|-1+\left|M_{2}\right|-1-\left(\left|M_{1} \cup M_{2}\right|-1\right) \\
& =\operatorname{dim} \Pi\left(M_{1} \cap M_{2}\right)-1=\operatorname{dim} \Pi\left(M_{1} \cap M_{2}\right) .
\end{aligned}
$$


Доказанное равенство совместно с очевидным включением $\Pi\left(M_{1}\right) \cap \Pi\left(M_{2}\right) \supseteq \Pi\left(M_{1} \cap\right.$ $M_{2}$ ) завершает доказательство справедливости требуемого равенства (b) условия (4).

СлЕДСТВИЕ 1. Если подмножество точек $M \subset \mathbb{R}^{m}$ находится в сильно общем положсени, то оно находится в общем положении.

Лемма 2. Если подмножсество точек $M \subset \mathbb{R}^{m}$ находится в общем положеении и состоит не более чем из $[(m-1) / 2]+m+3$ точек, то оно находится в сильно общем положении.

ДоказАТЕЛЬСтво. Так как множество $M$ находится в обшем положении, то согласно лемме 1 при $k=2$ условие (SGP) вьполнено.

Если $k \geqslant 3$ и пересечение соответствующих плоскостей пусто, то условие (SGP) вьполнено. Рассмотрим теперь случай, когда пересечение всех плоскостей непусто. Тогда по условию (2) леммы 1 из центрированности плоскостей $\Pi\left(M_{i}\right)$ и $\left(M_{j}\right)$ вытекает неравенство $\left|M_{i}\right|+\left|M_{j}\right| \geqslant m+2$. Сложение 3 соответствуюших неравенств дает неравенство $2|M| \geqslant 2\left(\left|M_{1}\right|+\left|M_{2}\right|+\left|M_{3}\right|\right) \geqslant 3 m+6$, что противоречит условию.

ЗАмЕчАнИЕ 2 . В пространстве $\mathbb{R}^{m}$ легко построить $([(m-1) / 2]+m+4)$-точечное подмножество, которое находится в общем положении, но не находится в сильно общем положении.

ПрЕДЛОЖЕНИЕ 3. Если $\Pi_{1} \cap \Pi_{2}=\varnothing u B \notin \Pi_{1}+\Pi_{2}$, то плоскости $\Pi_{1} u \Pi_{2}+B$ также не пересекаются.

ЛЕмма 3. Для всякого конечного или счетного подмножсества точек $M \subset \mathbb{R}^{m}$, находящегося в сильно общем положении, для всякой точки $A \in \mathbb{R}^{m}$ и всякого положительного числа $\varepsilon>0$ существует такая точка $B \in \mathbb{R}^{m}$, что $\varrho(A, B)<\varepsilon$ и множество $M^{\prime}=M \cup B$ находится в сильно общем положении.

ДокАЗАТЕльство. Для любого конечного набора попарно дизъюнктных подмножеств $M_{1}, \ldots, M_{k-1}, M_{k} \subseteq M$ такого, что пересечение плоскостей $\Pi\left(M_{1}\right), \ldots$, $\Pi\left(M_{k-1}\right)$ непусто и плоскость $\Pi\left(M_{1}\right) \cap \cdots \cap \Pi\left(M_{k-1}\right)+\Pi\left(M_{k}\right)$ не совпадает со всем пространством $\mathbb{R}^{m}$, положим

$$
\Pi\left(M_{1}, \ldots, M_{k-1}, M_{k}\right)=\Pi\left(M_{1}\right) \cap \cdots \cap \Pi\left(M_{k-1}\right)+\Pi\left(M_{k}\right) .
$$

При этом мы не исключаем наборы с пустым множеством $M_{k}$ и в этом случае полагаем $\Pi\left(M_{1}, \ldots, M_{k-1}, \varnothing\right)=\Pi\left(M_{1}\right) \cap \cdots \cap \Pi\left(M_{k-1}\right)$.

Тогда счетное множество точек $M \subset \mathbb{R}^{m}$ определяет счетное число плоскостей вида $\Pi\left(M_{1}, \ldots, M_{k}\right)$, каждая из которых имеет размерность $\leqslant m-1$. По счетной теореме суммы для замкнутых множеств [9] объединение всех отмеченных плоскостей $(+)$ имеет размерность $\leqslant m-1$. Следовательно, указанное объединение не содержит никакого открытого в $\mathbb{R}^{m}$ подмножества [23]. В $\varepsilon$-окрестности точки $A$ выберем точку $B$, лежащую в дополнении к указанному объединению плоскостей.

Покажем, что если множество точек $M$ находилось в сильно общем положении, то и множество $M^{\prime}=M \cup B$ находится в сильно общем положении.

Пусть $M_{1}, \ldots, M_{k}$ - попарно дизъюнктные подмножества множества $M^{\prime}$. Если $M_{1} \cup \cdots \cup M_{k} \subseteq M$, то указанные множества удовлетворяют условию (SGP), так как 
множество точек $M$ находится в сильно общем положении. Если $B \in M_{1} \cup \cdots \cup M_{k}$, то без ограничения общности можно считать, что $B \in M_{k}$.

Если $M_{k}=\{B\}$, то $B \notin \Pi\left(M_{1}\right)$, поэтому выполнено условие ( $\left.\varnothing\right)$ из (SGP). Если же $M_{k}=\widetilde{M}_{k} \cup\{B\}$, то справедливо по крайней мере одно из следуюших условий:

$$
\begin{aligned}
& \Pi\left(M_{1}\right) \cap \cdots \cap \Pi\left(M_{k-1}\right)+\Pi\left(\widetilde{M}_{k}\right) \neq \mathbb{R}^{m} ; \\
& \Pi\left(M_{1}\right) \cap \cdots \cap \Pi\left(M_{k-1}\right)+\Pi\left(M_{k}\right)=\mathbb{R}^{m} .
\end{aligned}
$$

Если $\Pi\left(M_{1}\right) \cap \cdots \cap \Pi\left(M_{k-1}\right)=\varnothing$, то пересечение большего числа плоскостей тем более пусто, и, тем самым, вьполнено условие ( $\varnothing$ ) из (SGP). Поэтому всюду в последующем мы будем считать, что $\Pi\left(M_{1}\right) \cap \cdots \cap \Pi\left(M_{k-1}\right) \neq \varnothing$.

Покажем, что при вьполнении условия (1) плоскости $\Pi\left(M_{1}\right) \cap \cdots \cap \Pi\left(M_{k-1}\right)$ и $\Pi\left(M_{k}\right)$ имеют пустое пересечение, а значит, удовлетворяют условию (=) из (SGP). Если пустое пересечение имеют плоскости $\Pi\left(M_{1}\right) \cap \cdots \cap \Pi\left(M_{k-1}\right)$ и $\Pi\left(\widetilde{M}_{k}\right)$, то искомые плоскости дизъюнктны согласно предложению 3 . Если же плоскости $\Pi\left(M_{1}\right) \cap \cdots \cap$ $\Pi\left(M_{k-1}\right)$ и П $\left(\widetilde{M}_{k}\right)$ имеют непустое пересечение, то согласно предложению 1 имеет место неравенство

$$
\begin{aligned}
\operatorname{dim} \Pi\left(M_{1}\right) \cap \cdots \cap \Pi\left(M_{k-1}\right) \cap \Pi\left(\widetilde{M}_{k}\right) & \\
= & \operatorname{dim} \Pi\left(M_{1}\right) \cap \cdots \cap \Pi\left(M_{k-1}\right)+\operatorname{dim} \Pi\left(\widetilde{M}_{k}\right) \\
& \quad-\operatorname{dim}\left(\Pi\left(M_{1}\right) \cap \cdots \cap \Pi\left(M_{k-1}\right)+\Pi\left(\widetilde{M}_{k}\right)\right) \\
\geqslant & \operatorname{dim} \Pi\left(M_{1}\right) \cap \cdots \cap \Pi\left(M_{k-1}\right)+\operatorname{dim} \Pi\left(\widetilde{M}_{k}\right)-(m-1) .
\end{aligned}
$$

Согласно же равенству (=) из (SGP) имеет место равенство

$$
\begin{aligned}
\operatorname{dim} & \Pi\left(M_{1}\right) \cap \cdots \cap \Pi\left(M_{k-1}\right) \cap \Pi\left(\widetilde{M}_{k}\right) \\
& =\operatorname{dim} \Pi\left(M_{1}\right)+\cdots+\operatorname{dim} \Pi\left(\widetilde{M}_{k}\right)-m(k-1) \\
& =\operatorname{dim} \Pi\left(M_{1}\right) \cap \cdots \cap \Pi\left(M_{k-1}\right)+\operatorname{dim} \Pi\left(\widetilde{M}_{k}\right)-m .
\end{aligned}
$$

Полученное противоречие завершает доказательство искомой дизъюнктности.

Покажем, что из условия (2) вытекает справедливость условия (SGP).

Если $\Pi\left(M_{1}\right) \cap \cdots \cap \Pi\left(M_{k-1}\right) \cap \Pi\left(M_{k}\right)=\varnothing$, то вьполнено условие ( $\left.\varnothing\right)$ из (SGP).

Если же $\Pi\left(M_{1}\right) \cap \cdots \cap \Pi\left(M_{k-1}\right) \cap \Pi\left(M_{k}\right) \neq \varnothing$, то согласно преложению 1 имеет место равенство

$$
\begin{aligned}
\operatorname{dim} \Pi\left(M_{1}\right) \cap \cdots \cap \Pi\left(M_{k-1}\right) \cap \Pi\left(M_{k}\right) & =\operatorname{dim} \Pi\left(M_{1}\right) \cap \cdots \cap \Pi\left(M_{k-1}\right)+\operatorname{dim} \Pi\left(M_{k}\right) \\
& \quad-\operatorname{dim}\left(\Pi\left(M_{1}\right) \cap \cdots \cap \Pi\left(M_{k-1}\right)+\Pi\left(M_{k}\right)\right) \\
= & \operatorname{dim} \Pi\left(M_{1}\right) \cap \cdots \cap \Pi\left(M_{k-1}\right)+\operatorname{dim} \Pi\left(M_{k}\right)-m \\
= & \operatorname{dim} \Pi\left(M_{1}\right)+\cdots+\operatorname{dim} \Pi\left(M_{k-1}\right)-m(k-2)+\operatorname{dim} \Pi\left(M_{k}\right)-m .
\end{aligned}
$$

Полученное равенство завершает доказательство. 
ТЕОРемА 1. Для любого счетного семейства непрерывных отображсений $f_{i}: X_{i} \rightarrow \mathbb{R}^{m}, i=1,2, \ldots$, вполне регулярных пространств $X_{i}$ и любого счетного семейства $\left\{\omega_{i}\right\}_{i=1}^{\infty}$ открытых покрытий пространства $\mathbb{R}^{m}$ существует такое семейство непрерывных отображений $g_{i}: X_{i} \rightarrow \mathbb{R}^{m}, \quad i=1,2, \ldots$, что отображения $f_{i}$ u $g_{i}$ являются $\omega_{i}$-близкими и для любого конечного подмножества $K$ натуральных чисел справедливо неравенство

$$
\operatorname{dim} \bigcap_{i \in K} g_{i}\left(X_{i}\right) \leqslant \sum_{i \in K} \operatorname{dim} X_{i}-m(|K|-1)
$$

ДОКАЗАТЕЛЬСТВо. Без ограничения общности будем считать, что $\operatorname{dim} X_{i} \leqslant m$ для всех индексов $i$. Для всякого покрытия $\omega_{i}$ построим звездно вписанное покрытие $\lambda_{i}$, т.е. в покрытие $\omega_{i}$ вписаны звезды всех элементов покрытия $\lambda_{i}$.

Шаг первый. Пусть все пространства $X_{i}$ являются счетными полиэдрами в слабой топологии. Без ограничения обшности можно считать, что все элементы всех покрытий $\omega_{i}$ выпуклы. Для каждого индекса $i$ возьмем такое барищентрическое подразделение $\widetilde{X}_{i}$ полиэдра $X_{i}$, что образ всякого симплекса лежит в некотором элементе покрытия $\lambda_{i}$. По лемме 3 можно взять такую $\lambda_{i}$-аппроксимацию образов вершин полиэдров $\widetilde{X}_{i}, i=1,2, \ldots$, что объединение аппроксимаций образов всех вершин всех полиэдров находится в сильно общем положении. Далее продолжим $\lambda_{i}$-аппроксимацию образов вершин полиэдра, т.е. отображение $g_{i}^{0}: \widetilde{X}_{i}^{0} \rightarrow \mathbb{R}^{m}$ по линейности на весь полиэдр $\widetilde{X}_{i}$. Построенное отображение $g_{i}$ и первоначальное отображение $f_{i}$ являются $\omega_{i}$-близкими.

Возьмем теперь конечньй набор симплексов $\sigma_{i}^{n_{i}}$ - по одному в разных полиэдрах $\widetilde{X}_{i}$. Так как множество $M=\bigcup_{i} g_{i}^{0}\left(\sigma_{i}^{n_{i}, 0}\right)$ находится в сильно общем положении, то $\bigcap \Pi\left(g_{i}^{0}\left(\sigma_{i}^{n_{i}, 0}\right)\right)=\varnothing$, или $\operatorname{dim} \bigcap \Pi\left(g_{i}^{0}\left(\sigma_{i}^{n_{i}, 0}\right)\right)=\sum_{i \in K} n_{i}-m(|K|-1)$. Но так как $g_{i}\left(\sigma_{i}^{n_{i}}\right) \subset \Pi\left(g_{i}^{0}\left(\sigma_{i}^{n_{i}, 0}\right)\right)$, то

$$
\operatorname{dim} \bigcap g_{i}\left(\sigma_{i}^{n_{i}}\right) \leqslant \sum_{i \in K} n_{i}-m(|K|-1) \leqslant \sum_{i \in K} \operatorname{dim} X_{i}-m(|K|-1)
$$

Так как симплексы можно брать произвольным образом, то теорема о размерности счетного объединения замкнутых множеств доказьвает искомое неравенство.

Шаг второй. Без ограничения общности можно считать, что покрытие $\lambda_{i}$ является счетным локально конечным покрытием. Тогда $f_{i}^{-1} \lambda_{i}$ является счетньм локально конечным функционально открытьм покрытием пространства $X_{i}$. Следовательно, в покрытие $f_{i}^{-1} \lambda_{i}$ можно вписать счетное локально конечное функционально открытое покрытие $\mu_{i}$ кратности $\leqslant \operatorname{dim} X_{i}+1$. Существует [9, гл. $\left.4, \S 2\right]$ такое отображение $q_{i}: N_{\mu_{i}} \rightarrow \mathbb{R}^{m}$, что отображения $f_{i}$ и $q_{i}$ ○ $p_{i}$ являются $\lambda_{i}$-близкими, где $N_{\mu_{i}}$ это нерв покрытия $\mu_{i}$, а $p_{i}: X_{i} \rightarrow N_{\mu_{i}}$ - каноническое отображение. Далее отображения $q_{i}$ заменим на $\lambda_{i}$-аппроксимации $g_{i}$, которые построены на первом шаге.

Отметим, что на втором шаге можно предполагать пространство $X_{i}$ метризуемьм и сепарабельным, а обший случай сводится к этому с помощью факторизационной теоремы Пасынкова [40], [9, гл. 6, §4]. 


\section{§. Регулярно ветвящиеся отображения}

Для усиления теоремы Гуревича нам понадобится следуюший глубокий результат из теории "разделения" отображений [26].

Теорема ДРАнИшнИковА. Пусть $X$ и $Y$ это компактные метрические пространства $u \operatorname{dim} X \leqslant m-3, \operatorname{dim} Y \leqslant m-3$. Тогда для любых непрерывных отображсний $f: X \rightarrow \mathbb{R}^{m}$ u $g: Y \rightarrow \mathbb{R}^{m}$ и любого $\varepsilon>0$ существуют такие $\varepsilon$-аппроксимации $f^{\prime}: X \rightarrow \mathbb{R}^{m}$ и $g^{\prime}: Y \rightarrow \mathbb{R}^{m}$ отображений $f$ и $g$, что

$$
\operatorname{dim} f^{\prime}(X) \cap g^{\prime}(Y) \leqslant \operatorname{dim} X \times Y-m .
$$

СЛЕДСТВИЕ 2. Пусть $X$ и $Y$ это компактные метрические пространства, $\Pi \subseteq \mathbb{R}^{m}$ это плоскость $и \operatorname{dim} X \leqslant m-3, \operatorname{dim} Y \leqslant m-3, \operatorname{dim} X \times Y+\operatorname{dim} \Pi \leqslant 2 m-$ 1. Тогда для любых непрерывных отображений $f: X \rightarrow \mathbb{R}^{m}$ u $g: Y \rightarrow \mathbb{R}^{m} u$ любого $\varepsilon>0$ существуют такие в-аппроксимащии $f^{\prime \prime}: X \rightarrow \mathbb{R}^{m}$ u $g^{\prime \prime}: Y \rightarrow \mathbb{R}^{m}$ отображений $f$ и $g$, что $f^{\prime \prime}(X) \cap g^{\prime \prime}(Y) \cap \Pi=\varnothing$.

ДокАЗАТЕльство. Теорема Дранишникова нам потребуется в несколько более сильной формулировке. Именно, существуют такие $\varepsilon / 2$-апроксимации, что пересе-

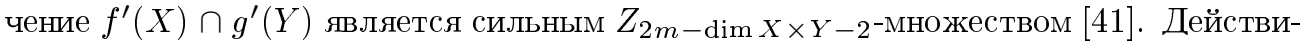
тельно, согласно доказательству Дранишникова компакт $f^{\prime}(X)$ является ручным, т.е. является $Z_{1}$-множеством. Если $m \leqslant 5$, то $\operatorname{dim} X+\operatorname{dim} Y \leqslant 2 m-6 \leqslant m-1$, поэтому согласно теореме 1 можно считать, что компакты $f^{\prime}(X)$ и $g^{\prime}(Y)$ дизъюнктны. Пусть $m \geqslant 6$. Согласно замечанию из работы Дранишникова [26] компакт $f^{\prime}(X) \cap g^{\prime}(Y)$ также является $Z_{1}$-множеством. При этом $\operatorname{dim} f^{\prime}(X) \cap g^{\prime}(Y) \leqslant \operatorname{dim} X \times Y-m$, поэтому согласно теореме Саммерхилла [41, предложение 2.1 , условие (iv)] компакт

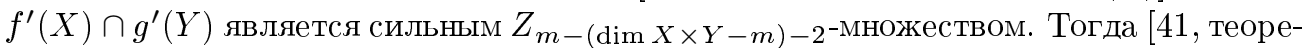
ма 1.1 и предложение 2.1, условие (i)] сушествует такой гомеоморфизм $h: \mathbb{R}^{m} \rightarrow \mathbb{R}^{m}$, что $\varrho(\operatorname{Id}, h)<\varepsilon / 2$ и $\operatorname{dim} h\left(f^{\prime}(X) \cap g^{\prime}(Y)\right) \cap \Pi \leqslant \operatorname{dim} X \times Y-m+\operatorname{dim} \Pi-m \leqslant-1$.

Отображения $f^{\prime \prime}=h \circ f^{\prime}$ и $g^{\prime \prime}=h \circ g^{\prime}$ являются искомыми $\varepsilon$-аппроксимациями.

Доказательство следующей теоремы повторяет доказательство теоремы Гуревича, предложенное в [25], с заменой ссылки на теорему 1 ссылкой на следствие 2.

Tеорема 2. Пусть $X$ это компактное метрическое пространство и $\operatorname{dim} X \leqslant$ $m-3$. Тогда множество $\mathscr{N}_{\operatorname{dim} X^{2}-m, 2}\left(X, \mathbb{R}^{m}\right)$ содержит всюду плотное $G_{\delta}-n о д-$ мно жество.

ЗАмечАниЕ 3. Результаты Е.В. Щепина [30] о кратных пересечениях позволяют и в общем случае усилить неравенство $\left(H_{k}\right)$.

\section{§ 3. Обращение теоремы Гуревича для полиэдров}

Через $B^{n}$ мы будем обозначать шар единичного радиуса в $n$-мерном евклидовом пространстве. В обращении теоремы Гуревича для полиэдров ключевую роль играет следуюшее обрашение теоремы о “разделении” отображений.

ПРЕДЛОЖЕНИЕ 4. Пусть $f_{1}: B^{n_{1}} \rightarrow \mathbb{R}^{m}$ u $f_{2}: B^{n_{2}} \rightarrow \mathbb{R}^{m}$ это такие невырожденные аффинные отображения, переводящие нулевую точку в нулевую, что

$$
\Pi\left(f\left(B^{n_{1}}\right)\right)+\Pi\left(f\left(B^{n_{2}}\right)\right)=\mathbb{R}^{m} .
$$


Тогда существует такое $\varepsilon>0$, что как только $\varrho\left(f_{i}, g_{i}\right)<\varepsilon, i=1,2$, так справедливо неравенство

$$
\operatorname{dim} g_{1}\left(B^{n_{1}}\right) \cap g_{2}\left(B^{n_{2}}\right) \geqslant n_{1}+n_{2}-m
$$

Лемма 4. Пусть $f_{1}: B^{n_{1}} \rightarrow \mathbb{R}^{m}, \ldots, f_{k+1}: B^{n_{k+1}} \rightarrow \mathbb{R}^{m}$ это такие невырожсденные аффинные отображения, переводящие нулевую точку в нулевую, что справедливо неравенство

$$
\operatorname{dim} \Pi\left(f_{1}\left(B^{n_{1}}\right)\right) \cap \cdots \cap \Pi\left(f_{k+1}\left(B^{n_{k+1}}\right)\right) \leqslant n_{1}+\cdots+n_{k+1}-m k .
$$

Тогда существует такое $\varepsilon>0$, что как только $\varrho\left(f_{i}, g_{i}\right)<\varepsilon, i=1, \ldots, k+1$, так для любого подмножества $K \subseteq\{1, \ldots, k+1\}$ справедливо неравенство

$$
\operatorname{dim} \bigcap_{i \in K} g_{i}\left(B^{n_{i}}\right) \geqslant \sum_{i \in K} n_{i}-m(|K|-1)
$$

ДоказАТельство. Доказательство разобьем на два шага.

Шаг первый. Пусть подмножество $K$ является множеством всех индексов. Так как плоскости, о которых говорится в лемме, пересекаются, то согласно предложению 3 можно считать, что в условии леммы вместо неравенства дано равенство. Данные отображения формулой

$$
F(\bar{x})=\left(f_{1} \times \cdots \times f_{k+1}\right)\left(x_{1}, \ldots, x_{k+1}\right)=\left(f_{1}\left(x_{1}\right), \ldots, f_{k+1}\left(x_{k+1}\right)\right)
$$

задают невырожденное аффинное отображение $F: B^{n_{1}+\cdots+n_{k+1}} \subset B^{n_{1}} \times \cdots \times B^{n_{k+1}}$ $\rightarrow \mathbb{R}^{m(k+1)}$. Рассмотрим также "диагональный" $m$-мерный куб

$$
\Delta\left(B^{m}\right)=\left\{\left(y_{1}, \ldots, y_{k+1}\right) \in\left(\mathbb{R}^{m}\right)^{k+1}: y_{1}=\cdots=y_{k+1} \in B^{m} \subset \mathbb{R}^{m}\right\}
$$

Плоскости $\Pi_{1}=\Pi\left(F\left(B^{n_{1}+\cdots+n_{k+1}}\right)\right)$ и $\Pi_{2}=\Delta$ имеют размерности $n_{1}+\cdots+n_{k+1}$ и $m$ соответственно. Из условия леммы несложно увидеть, что пересечение рассматриваемых плоскостей имеет размерность $n_{1}+\cdots+n_{k+1}-m k$. Тогда согласно предложению 2 их сумма имеет размерность $m(k+1)$, т.е. их сумма совпадает со всем пространством $\left(\mathbb{R}^{m}\right)^{k+1}$. $\mathrm{K}$ кубам, порождаюшим плоскости $\Pi_{1}$ и $\Pi_{2}$, применимо предложение 4. Поэтому сушествует такое $\varepsilon>0$, что никакое $\varepsilon$-шевеление отображения $F$ не уменьшает размерность пересечения образа с диагональю $\Delta$. Так как $\varepsilon$-шевеление отображений $f_{i}$ порождает $\sqrt{k+1} \varepsilon$-шевеление отображения $F$, то достаточно малое шевеление отображений $f_{i}$ не уменьшает размерность пересечения образа $G\left(B^{n_{1}+\cdots+n_{k+1}}\right)$ куба $B^{n_{1}+\cdots+n_{k+1}}$ с диагональю $\Delta$. Указанное же пересечение гомеоморфно пересечению образов $g_{i}\left(B^{n_{i}}\right)$ соответствуюших кубов $B^{n_{i}}$.

Второй шаг. Покажем, что для любого подмножества $K \subseteq\{1, \ldots, k+1\}$ вьполнены условия леммы. Доказательство проведем индукцией по числу $r=k+1-|K|$. При $r=0$ мы имеем в точности условие леммы, поэтому база индукции имеет место. 
Проведем теперь индуктивньй переход. Возьмем какой-нибудь индекс $j$ из дополнения к множеству $K$. Множество $\widetilde{K}=K \cup\{j\}$ имеет большую мошность, поэтому справедливо неравенство

$$
\operatorname{dim} \bigcap_{i \in \widetilde{K}} \Pi\left(f_{i}\left(B^{n_{i}}\right)\right) \leqslant \sum_{i \in K} n_{i}+n_{j}-m|K| .
$$

С другой стороны, согласно предложению 2 справедливо неравенство

$$
\begin{aligned}
& \operatorname{dim} \bigcap_{i \in K} \Pi\left(f_{i}\left(B^{n_{i}}\right)\right) \\
& =\operatorname{dim} \bigcap_{i \in \widetilde{K}} \Pi\left(f_{i}\left(B^{n_{i}}\right)\right)+\operatorname{dim}\left(\bigcap_{i \in K} \Pi\left(f_{i}\left(B^{n_{i}}\right)\right)+\Pi\left(f_{j}\left(B^{n_{j}}\right)\right)\right)-\operatorname{dim} \Pi\left(f_{j}\left(B^{n_{j}}\right)\right) \\
& \leqslant \sum_{i \in K} n_{i}+n_{j}-m|K|+m-n_{j}=\sum_{i \in K} n_{i}-m(|K|-1) .
\end{aligned}
$$

Итак, мы показали, что для любого семейства индексов справедливо условие леммы, тогда по уже сделанному первому шагу справедливо и заключение леммы. Отметим только, что в качестве “обшего” числа $\varepsilon$ нужно выбрать наименьшее из чисел по всем подмножествам индексов.

СлЕДСТВИЕ 3. Если неотрицательные числа $n_{1}, \ldots, n_{k+1}$, не превосходящие $m$, удовлетворяют неравенству

$$
n_{1}+\cdots+n_{k+1} \geqslant m k
$$

то существуют такие аффинные вложения $f_{1}: B^{n_{1}} \rightarrow \mathbb{R}^{m}, \ldots, f_{k+1}: B^{n_{k+1}} \rightarrow$ $\mathbb{R}^{m}$, переводящие нулевую точку в нулевую, что для некоторого $\varepsilon>0$ как толь$\kappa о \varrho\left(f_{i}, g_{i}\right)<\varepsilon, i=1, \ldots, k+1$, так для любого подмножества $K \subseteq\{1, \ldots, k+1\}$ справедливо неравенство

$$
\operatorname{dim} \bigcap_{i \in K} g_{i}\left(B^{n_{i}}\right) \geqslant \sum_{i \in K} n_{i}-m(|K|-1) .
$$

ДокАЗАТЕЛЬство. Рассмотрим в пространстве $\left(\mathbb{R}^{m}\right)^{k+1}$ базис

$$
e_{1}, \ldots, e_{m}, e_{1}, \ldots, e_{m}, \ldots, e_{1}, \ldots, e_{m}
$$

т.е. $k+1$ раз повторенный базис пространства $\mathbb{R}^{m}$. Полученные $m(k+1)$ векторы последовательно занумеруем. Теперь в качестве искомых вложений возьмем “тождественные" вложения куба $B^{n_{1}}$ - в подпространство, натянутое на первые $n_{1}$ векторов, куба $B^{n_{2}}$ в подпространство, натянутое на следующие $n_{2}$ векторов, и т. д.

Так как $0 \leqslant n_{i} \leqslant m$, то справедливы неравенства

$$
m \geqslant n_{1} \geqslant n_{1}+n_{2}-m \geqslant \cdots \geqslant n_{1}+\cdots+n_{k+1}-m k \geqslant 0 .
$$

Отсюда вытекает, что общей частью образов всех шаров является в точности шар, лежащий в евклидовом пространстве, натянутом на первые $n_{1}+\cdots+n_{k+1}-m k$ векторов. Следовательно, для взятых вложений шаров выполнены условия леммы 4 , что и доказывает справедливость утверждения следствия. 
СлЕДСТВИЕ 4. Если числа $n, m$ и $k$ удовлетворяют неравенству $n \geqslant(m-n) k$, то существуют такие непрерывное отображение $f: B^{n} \rightarrow \mathbb{R}^{m}$ и положстельное число $\varepsilon>0$, что как только $g: B^{n} \rightarrow \mathbb{R}^{m}$ это такое непрерывное отображение, что $\varrho(f, g)<\varepsilon$, так $\operatorname{dim} B_{k+1}(g) \geqslant n-(m-n) k$, в частности, существует точка $y \in \mathbb{R}^{m}$, полный прообраз которой состоит не менее чем из $k+1$ различной точки.

ДокаЗАТЕЛЬСтво. Рассмотрим в шаре $B^{n} k+1$ попарно дизъюнктных шаров радиуса $\delta: B_{1}^{n}, \ldots, B_{k+1}^{n}$. Из неравенства $n \geqslant(m-n) k$ легко вьвести, что размерности шаров удовлетворяют условию следствия 3. Следовательно, существуют такие вложения $f_{1}: B_{1}^{n} \rightarrow \mathbb{R}^{m}, \ldots, f_{k+1}: B_{k+1}^{n} \rightarrow \mathbb{R}^{m}$, что для достаточно близких отображений $g_{1}: B_{1}^{n} \rightarrow \mathbb{R}^{m}, \ldots, g_{k+1}: B_{k+1}^{n} \rightarrow \mathbb{R}^{m}$ имеет место неравенство

$$
\operatorname{dim} \bigcap_{i=1}^{k+1} g_{i}\left(B_{i}^{n}\right) \geqslant n(k+1)-m k=n-(m-n) k \geqslant 0
$$

Последнее неравенство означает, в частности, что если отображения $g_{i}$ достаточно близки к отображениям $f_{i}$ при $i=1, \ldots, k+1$, то множество $\bigcap_{i=1}^{k+1} g_{i}\left(B_{i}^{n}\right)$ не пусто.

Объединение шаров $B_{i}^{n}$ замкнуто в шаре $B^{n}$, поэтому из экстензорных свойств пространства $\mathbb{R}^{m}$ следует, что построенные отображения можно продолжить непрерьвно на весь шар $B^{n}$ до такого отображения $f: B^{n} \rightarrow \mathbb{R}^{m}$, что $\left.f\right|_{B_{i}^{n}}=f_{i}$. Отображение $f$ является искомым.

СлЕДСТВИЕ 5. Для компактного полиэдра $P$ c $\operatorname{dim} P \leqslant m$ u чельхх чисел $r \geqslant$ -1 и $k \geqslant 0$ следующие условия эквивалентны.

(1) Имеет место неравенство $\operatorname{dim} P-r \leqslant(m-\operatorname{dim} P) k$.

(2) Множсество $\mathscr{N}_{r, k+1}\left(P, \mathbb{R}^{m}\right)$ всюду плотно в пространстве $\mathscr{C}\left(P, \mathbb{R}^{m}\right)$.

ДокАЗАТЕльство. Импликация $(1) \Rightarrow(2)$ содержится в теореме Гуревича.

Так как всякий полиэдр $P$ содержит замкнутый диск $B^{\operatorname{dim} P}$ и всякое отображение с диска может быть продолжено на весь полиэдр $P$, то импликашия $(2) \Rightarrow(1)$ вытекает из следствия 4.

СЛЕДСТВИЕ 6 . Для компактного полиэдра $P$ следующие условия әквивалент$H b l$.

(1) Имеет место неравенство $\operatorname{dim} P+1 \leqslant(m-\operatorname{dim} P) k$.

(2) Множество всех не более чем $k$-кратных отображений полиэдра $P$ в m-мерное евклидово пространство $\mathbb{R}^{m}$ всюду плотно в пространстве $\mathscr{C}\left(P, \mathbb{R}^{m}\right)$.

Пусть $\Delta_{n}^{N}$ это $n$-мерньй остов $N$-мерного симплекса.

ТЕОРема 3. При $N=(m+1)(q-1)$ для всякого линейного отображения $f: \Delta_{m}^{N} \rightarrow \mathbb{R}^{m}$, у которого образь вершин находятся в сильно общем положении, существует такое $\varepsilon>0$, что для всякого непрерывного отображсения $g: \Delta_{m}^{N} \rightarrow \mathbb{R}^{m}$ такого, что $\varrho(f, g)<\varepsilon$, существуют такие попарно дизбюнктные замкнутые симплексы $\sigma_{1}, \ldots, \sigma_{q} \subset \Delta_{m}^{N}$, ито $g\left(\sigma_{1}\right) \cap \cdots \cap g\left(\sigma_{q}\right) \neq \varnothing$. 
ДоКАЗАТЕльство. Продолжим отображение $f$ по линейности на весь симплекс $F: \Delta^{N} \rightarrow \mathbb{R}^{m}$. Согласно теореме Тверберг [32] существуют такие попарно дизбюнктные замкнутые симплексы $\sigma_{1}, \ldots, \sigma_{q} \subset \Delta^{N}$, что $F\left(\sigma_{1}\right) \cap \cdots \cap F\left(\sigma_{q}\right) \neq \varnothing$.

Прежде всего, покажем, что в условиях теоремы все симплексы $\sigma_{i}, i=1, \ldots, q$, не более чем $m$-мерны. Если $\operatorname{dim} \sigma_{q} \geqslant m+1$, то в симплексе $\sigma_{q}$ не менее $m+2$ вершин. Из попарной дизъюнктности симплексов вытекает, что в оставшихся симплексах не больше $N+1-(m+2)=(m+1)(q-2)$ вершин. Следовательно,

$$
\operatorname{dim} \sigma_{1}+\cdots+\operatorname{dim} \sigma_{q-1} \leqslant(m+1)(q-2)-(q-1)=m(q-2)-1 .
$$

Так как $F\left(\sigma_{1}\right) \cap \cdots \cap F\left(\sigma_{q}\right) \neq \varnothing$, то $\Pi\left(F\left(\sigma_{1}\right)\right) \cap \cdots \cap \Pi\left(F\left(\sigma_{q}\right)\right) \neq \varnothing$, поэтому из условия сильно общего положения вытекает неравенство

$$
\operatorname{dim} \Pi\left(F\left(\sigma_{1}\right)\right) \cap \cdots \cap \Pi\left(F\left(\sigma_{q}\right)\right) \leqslant m(q-2)-1+m-m(q-1)=-1 .
$$

Полученное неравенство противоречит центрированности рассматриваемых плоскостей. Итак, мы доказали, что $\operatorname{dim} \sigma_{i} \leqslant m$ при $i=1, \ldots, q$.

Кроме того, вьшеприведенное рассуждение показывает, что симплексы $\sigma_{1}, \ldots, \sigma_{q}$ должны содержать все вершины симплекса $\Delta^{N}$, т.е. $\operatorname{dim} \sigma_{1}+\cdots+\operatorname{dim} \sigma_{q}=m(q-1)$. Отсюда, в частности, вытекает, что, если хоть один из симплексов $\sigma_{i}, i=1, \ldots, q$, заменить на собственную грань, то образы получившегося набора симплексов не имеют общей точки. Это означает, что во внутренности каждого симплекса $\sigma_{i}, i=1, \ldots, q$, имеется такая точка $x_{i}$, что $f\left(x_{1}\right)=\cdots=f\left(x_{q}\right)$. В каждом из симплексов $\sigma_{i}$ возьмем $\operatorname{dim} \sigma_{i}$-мерный шар $B_{i}$ с центром в точке $x_{i}$ (и некоторым положительным радиусом при $\left.\operatorname{dim} \sigma_{i} \geqslant 1\right)$. Шары $B_{i}$ и их афффинные вложения $f_{i}=\left.f\right|_{B_{i}}$ удовлетворяют условию леммы 4 , которая и гарантирует существование искомого числа $\varepsilon>0$.

Доказанная теорема позволяет дать следуюший вариант теоремы Тверберг, которьй, впрочем, легко получается из теоремы Тверберг и с помошью теоремы Каратеодори.

СлЕДСТВИЕ 7. При $N=(m+1)(q-1)$ для всякого линейного отображения $f: \Delta_{m}^{N} \rightarrow \mathbb{R}^{m}$ существуют такие попарно дизбюнктные замкнутые симплексь $\sigma_{1}, \ldots, \sigma_{q} \subset \Delta_{m}^{N}$, ито $f\left(\sigma_{1}\right) \cap \cdots \cap f\left(\sigma_{q}\right) \neq \varnothing$.

ДоказАТЕЛьство. Отображения $f: \Delta_{n}^{N} \rightarrow \mathbb{R}^{m}$, для которых существует $q$ штук попарно дизъюнктных замкнутых симплексов с центрированньми образами, образуют замкнутое множество. Согласно же лемме 3 любое линейное отображение $f: \Delta_{n}^{N} \rightarrow \mathbb{R}^{m}$ лежит в замыкании линейных отображений, у которых образы вершин находятся в сильно общем положении.

ЗАмечАниЕ 4. Доказательство теоремы 3 показывает, что любые $(m+1)(q-1)$ точек в $\mathbb{R}^{m}$, находяшихся в сильно общем положении, задают линейное отображение $f: \Delta^{(m+1)(q-1)-1} \rightarrow \mathbb{R}^{m}$, для которого утверждение теоремы Тверберг неверно. $\mathrm{X}$. Тверберг показьвает невозможность уменьшения числа $N$ с помощью более сильного понятия общего положения - все координаты всех точек алгебраически независимы. При $q=2$ теорема Тверберг преврашается в классическую теорему Радона [42]. 
ЗАмечАниЕ 5 . При $q=2, q$ - простое и $q$ - степень простого, как показали Бажмози и Барани [43], Барани, Шлоссман и Сюч [44] и Воловиков [45] соответственно, теорема Тверберг справедлива для любого непрерьвного отображения $g: \Delta^{N} \rightarrow \mathbb{R}^{m}$, где $N=(m+1)(q-1)$. Случай произвольного q остается открытым. Так как в любой окрестности тривиального (линейного) отображения $f: \Delta_{n}^{N} \rightarrow \overline{0} \in \mathbb{R}^{m}$ содержится отображение, гомотетичное любому заранее заданному непрерьвному отображению, то справедливость теоремы 3 для произвольных линейных отображений равносильна справедливости теоремы Тверберг для произвольных непрерывных отображений. Однако здесь необходимо отметить, что в топологических вариантах теоремы Тверберг, т.е. в случае непрерывного отображения $f: \Delta^{N} \rightarrow \mathbb{R}^{m}$, на размерность симплексов $\sigma_{1}, \ldots, \sigma_{q} \subset \Delta^{N}$ не удается наложить никакого ограничения, кроме очевидной оценки для числа вершин семейства попарно дизъюнктных симплексов: $\operatorname{dim} \sigma_{1}+\cdots+\operatorname{dim} \sigma_{q} \leqslant N+1-q$. В работах ван Кампена и Флореса, Саркариа и Воловикова (для $q=2, q$ - простое число и $q$ - степень простого числа) получены глубокие результаты о существовании в $\Delta_{n}^{N}$ попарно дизъюнктных симплексов с центрированными образами, но уже для $N=(m+2)(q-1)$ или $N=(n+2) q-2$ (при справедливости отрицания неравенства $(n, m, q-1))$. Приложению подобных результатов к обрашению теоремы Гуревича посвяшен наш пятый параграф.

\section{$\S$ 4. Проблемы Гуревича и Нёбелинга}

В работе [22] В. Гуревич выдвинул следуюшую гипотезу. "Кажется очень вероятным, что компакт $X$ имеет размерность $\leqslant n$ тогда и только тогда, когда множество $\mathscr{E}\left(X, \mathbb{R}^{2 n+1}\right)$ всех вложений $X$ в $\mathbb{R}^{2 n+1}$ (т.е. множество $\left.\mathscr{N}_{-1,2}\left(X, \mathbb{R}^{2 n+1}\right)\right)$ всюду плотно в пространстве $\mathscr{C}\left(X, \mathbb{R}^{2 n+1}\right)$."

Теорема ДРАНИШНИКОВА-ВеСтА. Если для компактов $X$ и Y имеет место неравенство $\operatorname{dim} X \times Y \geqslant m$, то существуют такие непрерывные отображсения

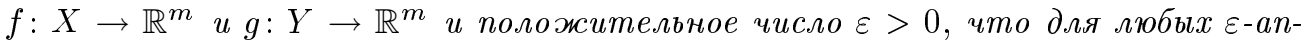
проксимаций $f_{\varepsilon}$ и $g_{\varepsilon} f_{\varepsilon}(X) \cap g_{\varepsilon}(Y) \neq \varnothing$.

Следующее утверждение хорошо известно, но так как во всех опубликованных формулировках имеется дополнительное условие $m=2 \operatorname{dim} X$, то мы приводим его доказательство.

СЛЕДСТВИЕ ДРАНИШНИКОВА-ВеСТА. Если для компакта $X$ множество $\mathscr{E}\left(X, \mathbb{R}^{m}\right)$ всех вложсений $X$ в $\mathbb{R}^{m}$ всюду плотно в пространстве $\mathscr{C}\left(X, \mathbb{R}^{m}\right)$, то имеет место неравенство $\operatorname{dim} X^{2} \leqslant m-1$.

ДокАЗАтельство. Пусть $\operatorname{dim} X^{2} \geqslant m$. Тогда в пространстве $X$ сушествуют такие дизъюнктные подкомпакты $X_{1}$ и $X_{2}$, что $\operatorname{dim} X_{1} \times X_{2} \geqslant m$ [25, с. 251]. Возьмем отображения $f_{1}: X_{1} \rightarrow \mathbb{R}^{n+1}$ и $f_{2}: X_{2} \rightarrow \mathbb{R}^{n+1}$, о которых говорится в теореме Дранишникова-Веста. Тогда для любых достаточно близких отображений образы компактов $X_{1}$ и $X_{2}$ пересекаются. Продолжим отображение $f_{1} \cup f_{2}$ с компакта $X_{1} \cup X_{2}$ на все пространство $X$. Построенное отображение не есть равномерньй предел вложений.

Доказательство гипотезы Гуревича легко вытекает из сформулированного следствия и формулы для вычисления размерности степеней компакта [28]. 
ТЕОРемА БОКШТЕЙНА-КУЗЬМИНОВА. ДЛя любого компакта X размерности его степеней вычисляются по одному из следующих законов:

(1) $\operatorname{dim} X^{k}=k \cdot \operatorname{dim} X$ для всех $k \geqslant 1$;

(2) $\operatorname{dim} X^{k}=k \cdot(\operatorname{dim} X-1)+1 \partial$ дя все $k \geqslant 1$.

СлЕДСТВИЕ 8. Для компакта X следующие условия әквивалентны.

(1) Имеет место неравенство $\operatorname{dim} X \leqslant n$.

(2) Множество $\mathscr{E}\left(X, \mathbb{R}^{2 n+1}\right)$ всех вложсений $X$ в $\mathbb{R}^{2 n+1}$ всюду плотно в пространстве $\mathscr{C}\left(X, \mathbb{R}^{2 n+1}\right)$.

ДокАЗАТЕльство. Импликащия $(1) \Rightarrow(2)$ доказана Гуревичем.

$(2) \Rightarrow(1)$. Согласно следствию Дранишникова-Веста из условия (2) вытекает неравенство $\operatorname{dim} X^{2} \leqslant 2 n$. С другой стороны, по теореме Бокштейна-Кузьминова справедливо неравенство $2 \operatorname{dim} X-1 \leqslant \operatorname{dim} X^{2}$. Два вьписанных неравенства дают неравенство $\operatorname{dim} X \leqslant n+1 / 2$, которое эквивалентно неравенству условия (1).

Гипотезу Нёбелинга [27] можно сформулировать следующим образом. "Вероятно, компактное пространство $X$ имеет размерность $\leqslant n$ тогда и только тогда, когда для некоторого $m$ такого, что $n+1 \leqslant m \leqslant 2 n+1$, конечнократные отображения из $\mathscr{N}_{2 n-m, 2}\left(X, \mathbb{R}^{m}\right)$ образуют всюду плотное подмножество $\mathscr{C}\left(X, \mathbb{R}^{m}\right)$ ".

ТЕОРемА ДРАНИШНИКОВА-РЕПОВША-ШЕПИНА. Для любъ $Y$ и всякого $m$ существуют такие непрерывные отображения $f: X \rightarrow \mathbb{R}^{m} u$ $g: Y \rightarrow \mathbb{R}^{m}$ и положмительное число $\varepsilon>0$, что для любых в-аппроксимаций $f_{\varepsilon} u$ $g_{\varepsilon}$ справедливо неравенство

$$
\operatorname{dim} f_{\varepsilon}(X) \cap g_{\varepsilon}(Y) \geqslant \operatorname{dim} X \times Y-m
$$

СлЕДСТВИЕ 9. Для компакта $X$ и целых чисел $n$ u $m$ maкux, что $n+1 \leqslant m \leqslant$ $2 n+1$, следующие условия эквивалентны.

(1) Имеет место неравенство $\operatorname{dim} X \leqslant n$.

(2) Множество $\mathscr{N}_{2 n-m, 2}\left(X, \mathbb{R}^{m}\right)$ всюду плотно в пространстве $\mathscr{C}\left(X, \mathbb{R}^{m}\right)$.

ДокАЗАТЕльСТво. Импликация $(1) \Rightarrow(2)$ доказана Гуревичем.

$(2) \Rightarrow(1)$. В пространстве $X$ существуют такие два дизъюнктных компакта $X_{1}$ и $X_{2}$, что $\operatorname{dim} X_{1} \times X_{2}=\operatorname{dim} X^{2}$. По теореме Дранишникова-Реповша-Шепина сушествуют такие отображения $f_{i}: X_{i} \rightarrow \mathbb{R}^{m}, i=1,2$, и такое положительное число $\varepsilon$, что как только $\varrho\left(f_{i}, g_{i}\right)<\varepsilon, i=1,2$, где $g_{i}: X_{i} \rightarrow \mathbb{R}^{m}$ это непрерывные отображения, так

$$
\operatorname{dim} g_{1}\left(X_{1}\right) \cap g_{2}\left(X_{2}\right) \geqslant \operatorname{dim} X_{1} \times X_{2}-m=\operatorname{dim} X^{2}-m .
$$

Продолжим теперь непрерывное отображение $f_{1} \cup f_{2}: X_{1} \cup X_{2} \rightarrow \mathbb{R}^{m}$ до непрерьвного отображения $f: X \rightarrow \mathbb{R}^{m}$. Всякая $\varepsilon$-аппроксимация $g \in \mathscr{N}_{2 n-m, 2}\left(X, \mathbb{R}^{m}\right)$ отображения $f$ порождает $\varepsilon$-аппроксимации $g_{i}=g \mid X_{i}$ отображений $f_{i}$. При этом из включения $g_{1}\left(X_{1}\right) \cap g_{2}\left(X_{2}\right) \subseteq B_{2}(g)$ и условия теоремы вытекает неравенство

$$
\operatorname{dim} X^{2}-m \leqslant \operatorname{dim} g_{1}\left(X_{1}\right) \cap g_{2}\left(X_{2}\right) \leqslant \operatorname{dim} B_{2}(g) \leqslant 2 n-m .
$$


Полученное неравенство эквивалентно неравенству $\operatorname{dim} X^{2} \leqslant 2 n$. С другой стороны, неравенство Бокштейна-Кузьминова $2 \operatorname{dim} X-1 \leqslant \operatorname{dim} X^{2}$ приводит к неравенству $2 \operatorname{dim} X \leqslant 2 n+1$, т.е. к неравенству $\operatorname{dim} X \leqslant n+1 / 2$. Целочисленность размерности влечет искомое неравенство.

\section{§. Обращение теоремы существования для полиэдров}

Для доказательства теоремы, обратной к теореме существования “экономичного" отображения, нам понадобятся некоторые факты из теории индекса свободного действия конечной групш $G$. По всей видимости, в настоящее время относительно полно построена теория индекса только для групшы $G=\left(\mathbb{Z}_{p}\right)^{\alpha}$, где $p$ это некоторое простое число, а $\alpha \geqslant 1$. Случай грушы $G=\mathbb{Z}_{p}$ исследован в работах многих авторов и является уже почти классическим, случай же обшей группы $G=\left(\mathbb{Z}_{p}\right)^{\alpha}$ исследован в основном в работах А. Ю. Воловикова [20]. Приведем некоторые, необходимые в дальнейшем, понятия и факты.

Пусть $K$ это симплициальньй комплекс. Определим комплекс $P^{q}(K) \subset K^{q}$ как объединение таких произведений $\sigma_{1} \times \cdots \times \sigma_{q}$ симплексов из $K$, что симплексы $\sigma_{1}, \ldots, \sigma_{q} \subset K$ являются попарно дизъюнктными.

Симметрическая группа $\Sigma_{q}$ действует на полиэдре $K^{q}$ и на его подполиэдре $P^{q}(K)$ перестановками координат. Если $q=p^{\alpha}$ для некоторого числа $p$, то на пространстве $K^{q}$ и его подпространстве $P^{q}(K)$ действует и группа $\left(\mathbb{Z}_{p}\right)^{\alpha}$. В этом случае на пространстве $P^{q}(K)$ действие группы $\left(\mathbb{Z}_{p}\right)^{\alpha}$ свободное, поэтому определен индекс

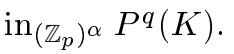

ТЕОРемА ВоловИКОВА. Для простого числа $p$ и натурального $\alpha \geqslant 1$ при $N \leqslant(n+2) q-2$, где $q=p^{\alpha}$, справедливо неравенство

$$
\operatorname{in}_{\left(\mathbb{Z}_{p}\right)^{\alpha}} P^{q}\left(\Delta_{n}^{N}\right) \geqslant N-2(q-1) .
$$

Эта теорема есть частный случай вьчислений Воловикова: в работе [36] нужно положить $n=\alpha, s=n+1, j=2$ и $k=0$.

Обозначим через $p[q]$ наименьшее простое число, большее $q-1$.

ТЕОремА 4. Пусть числа $n, m, q$ и $N$ удовлетворяют неравенствам

$$
(m+1)(p[q]-1)+q-1 \leqslant N \leqslant(n+2) p[q]-2 .
$$

Тогда для всякого непрерывного отображения $f: \Delta_{n}^{N} \rightarrow \mathbb{R}^{m}$ существуют такие попарно дизбюнктные замкнутые симплексы $\sigma_{1}, \ldots, \sigma_{q} \subset \Delta_{n}^{N}$, что пересечение их образов непусто $f\left(\sigma_{1}\right) \cap \cdots \cap f\left(\sigma_{q}\right) \neq \varnothing$.

ДокАЗАТЕльство. Отображение $f: \Delta_{n}^{N} \rightarrow \mathbb{R}^{m}$ по формуле $\tilde{f}=f \circ \pi_{1}$, т.е. формуле $\widetilde{f}\left(x_{1}, \ldots, x_{p[q]}\right)=f\left(x_{1}\right)$, порождает отображение

$$
\tilde{f}: P^{p[q]}\left(\Delta_{n}^{N}\right) \rightarrow \mathbb{R}^{m}
$$

Согласно теореме Воловикова имеет место неравенство

$$
\operatorname{in}_{\mathbb{Z}_{p[q]}} P^{p[q]}\left(\Delta_{n}^{N}\right) \geqslant N-2(p[q]-1) \geqslant(m-1)(p[q]-1)+q-1 .
$$


Так как согласно теореме Чебьшёва справедливо неравенство $p[q] \leqslant 2 q-1$, то к отображению $\widetilde{f}$ применима теорема Коэна-Ласка о частичной склейке орбиты $[37$, теорема 4]. Следовательно, существует такая точка $x \in P^{p[q]}\left(\Delta_{n}^{N}\right)$, что по крайней мере $q$ точек ее орбиты отображаются в одну точку. Склеиваемые точки орбиты и задают $q$ штук искомых попарно дизъюнктных симплексов, образы которых имеют непустое пересечение.

СлЕДСТВИЕ 10. Если при $N=(n+2) p[q]-2($ или $N=(m+1)(p[q]-1)+q-1)$ существует не более чем $(q-1)$-кратное отображсение $f: \Delta_{n}^{N} \rightarrow \mathbb{R}^{m}$, то справедливо неравенство

$$
n+1 \leqslant(m-n)(q-1)+(m-n-1)(p[q]-q) .
$$

ДокАЗАТЕльство. Предположим, что справедливо обратное неравенство

$$
(m-n)(q-1)+(m-n-1)(p[q]-q) \leqslant n,
$$

т.е. неравенство

$$
(m+1)(p[q]-1)+q-1 \leqslant(n+2) p[q]-2 .
$$

Тогда при любом из данных в условии следствия чисел $N$ применима теорема 4 . Согласно теореме 4 для любого отображения $\Delta_{n}^{N}$ в $\mathbb{R}^{m}$ сушествуют $q$ штук попарно непересекаюшихся замкнутых симплексов $\sigma_{1}, \ldots, \sigma_{q}$ с непустым пересечением их образов. Тогда понятно, что прообраз любой точки из этого пересечения $f\left(\sigma_{1}\right) \cap \cdots \cap f\left(\sigma_{q}\right)$ содержит точки в каждом из симплексов $\sigma_{1}, \ldots, \sigma_{q}$, а значит, имеет мошность не менее $q$, что противоречит условию.

ЗАмЕчАНИЕ 6 . При $p[q]=q$, т.е. в случае, когда число $q=k+1$ является простым, мы получаем в точности теорему Саркариа [35] и, в частности, обрашение теоремы сушествования “экономичного” отображения на классе всех $n$-мерных полиэдров. Обрашение теоремы сушествования “экономичного” отображения получается из следствия 10 и при некоторых не простых значениях числа $k+1$, но перед проведением соответствующего анализа приведем один общий случай.

СЛЕДСТВИЕ 11. При $N=(n+2) p[n+1]-2$ для всякого непрерывного отобрахсения $f: \Delta_{n}^{N} \rightarrow \mathbb{R}^{n+1}$ существуют такие попарно дизбюнктные замкнутые симплексы $\sigma_{1}, \ldots, \sigma_{n+1} \subset \Delta_{n}^{N}$, что пересечение их образов непусто.

ДокАЗАТЕльство. Для чисел $n, m=n+1, q=n+1$ и числа $N=(n+2) p[n+1]-2$ справедливы неравенства теоремы 4 , поэтому сушествование искомых симплексов гарантируется указанной теоремой.

Неравенство из следствия 10 превращается в неравенство $(n, m, q-1)$ (достаточное условие в следствии Гуревича о существовании “экономичного” отображения) при простом числе $q$ (замечание 6) и при $m=n+1$ (следствие 11). Однако за счет того, что все числа в неравенстве следствия 10 являются иельми числами, из этого формально более слабого неравенства неравенство $(n, m, q-1)$ вытекает и в некоторых других случаях. Так как для разбора всех случаев, для которых справедливо обрашение следствия Гуревича, мы не подготовили почву (нужно было сводить все 
к теореме о частичной склейке орбиты группы $\left(\mathbb{Z}_{p}\right)^{\alpha}$, а не групы $\mathbb{Z}_{p}$, как в теореме Коэна-Ласка), то здесь мы опишем только самьй первьй случай (при этом все три числа $n, m$ и $q$ одновременно принимают минимальное значение) среди всех случаев, остаюшихся к настоящему времени открытыми.

Итак, пусть числа $n, m$ и $q$ удовлетворяют неравенству следствия 10 и не удовлетворяют неравенству $(n, m, q-1)$, т.е. удовлетворяют системе неравенств

$$
\left\{\begin{array}{l}
n+1 \leqslant(m-n)(q-1)+(m-n-1)(p[q]-q), \\
(m-n)(q-1) \leqslant n .
\end{array}\right.
$$

Обозначим число $p[q]-q$ через $\delta$ и приведем в каждом неравенстве подобные члены

$$
\left\{\begin{array}{l}
n(q+\delta)+1+\delta \leqslant m(q-1+\delta) \\
m(q-1) \leqslant n q
\end{array}\right.
$$

Полученную систему можно переписать в следующем виде:

$$
n(q+\delta)(q-1)+(1+\delta)(q-1) \leqslant m(q-1+\delta)(q-1) \leqslant n q(q-1+\delta) .
$$

Крайние члены полученной цепочки неравенств приводят к неравенству

$$
(1+\delta)(q-1) \leqslant \delta n,
$$

т.е., при $\delta \neq 0$, к неравенству

$$
(1+1 / \delta)(q-1) \leqslant n .
$$

При $\delta=0$, т.е. при простом числе $q=2,3,5,7,11,13, \ldots$, обрашение следствия Гуревича справедливо согласно теореме Саркариа (замечание 6).

При $q=4,8,9, \ldots$ число $\delta \neq 0$, но обращение следствия Гуревича имеет место по теореме Воловикова [36], которая есть следствие сформулированной вьше теоремы Воловикова и другой теоремы Воловикова $[20]$ о полной склейке $\left(\mathbb{Z}_{p}\right)^{\alpha}$-орбиты.

При $\delta=1$, т.е. при $q=6,10,12, \ldots$, неравенство на $n$ приобретает вид $2(q-1) \leqslant n$. При $n=2(q-1)+\beta$, где $\beta \geqslant 0$ - целое число, на число $m$ получается система неравенств $2 q+\beta+\beta / q \leqslant m \leqslant 2 q+\beta+\beta /(q-1)$. В некоторых случаях полученное неравенство не имеет целых решений (относительно $m$ ), т.е. при некоторых числах $n$ $(\beta)$ и $q$ обрашение следствия Гуревича справедливо для всех $m$. Так как мы ищем "маломерньй" открытый случай, то полагаем $n=2 q-2$ и получаем $m=2 q$. Так как при $\delta=1$ наименьшее значение $q=6$ и соответственно $n=10$ и $m=12$, то найдена тройка, соответствуюшая наименьшему значению $q$, из всех троек $\{n, m, q\}$, для которых вопрос справедливости обрашения следствия Гуревича открыт.

Покажем, что для больших значений $q$ значения чисел $n$ и $m$ также больше. При $\delta \geqslant 2$, т.е. при $q=14, \ldots$, справедливо неравенство $14 \leqslant q \leqslant(1+1 / \delta)(q-1) \leqslant n$.

Резюмируем наш анализ в виде следуюшего вопроса: "Всякий ли 10 -мерный компакт (полиэдр) (например, $\Delta_{10}^{70}$ ) можно не более чем 5-кратно отобразить $\theta \mathbb{R}^{12}$ ?" 
ЗАмечАниЕ 7. Теорема ван Кампена-Флореса о невложимости джойнов полиэдров $\Delta_{n}^{2 n+2}$ позволяет оценивать снизу размерность евклидова пространства, в которое может быть $k$-регулярно [46] вложен полиэдр $\Delta_{n}^{N}$.

Если пространство $\Delta_{n}^{N}$, где $N=n k+n+k+\frac{k+1}{2}$, моэкно $k$-регулярно, где $k=2 l-1$, вложить в $\mathbb{R}^{m}$, то $m \geqslant n k+n+k$.

Если пространство $\Delta_{n}^{N}$, где $N=n k+k+\max \left\{\frac{k-2}{2}, 1\right\}$, можнно $k$-регулярно, где $k=2 l$, влоэиить в $\mathbb{R}^{m}$, то $m \geqslant n k+k$.

Вместе с теоремой В.Г. Болтянского [47] о плотности при $m \geqslant k \operatorname{dim} X+\operatorname{dim} X+k$ множества всех $k$-регулярных вложений компакта $X$ в $\mathbb{R}^{m}$ наша оценка снизу дает для нечетных чисел $k$ решение задачи Борсука-Болтянского определения размерности евклидова пространства, в которое может быть $k$-регулярно вложен всякий компакт (полиэдр) размерности $n$.

\section{§. Когомологическая размерность}

Будем говорить, что алгебраический дефект размерной неполноченности A-def $X$ конечномерного компакта $X$ равен $k$, если для всякой абелевой групшы $G$ справедливо неравенство

$$
\operatorname{dim}_{G} X \geqslant \operatorname{dim} X-k
$$

причем для некоторой групшы имеет место равенство.

Будем говорить, что геометрический дефект размерной неполноченности G-def $X$ конечномерного компакта $X$ равен $k$, если для всякого конечномерного компакта $Y$ справедливо неравенство

$$
\operatorname{dim} X \times Y \geqslant \operatorname{dim} X+\operatorname{dim} Y-k
$$

причем для некоторого компакта имеет место равенство.

Так как для компактного пространства $X$ для любой абелевой группы $G$ справедливо неравенство $\operatorname{dim}_{G} X \leqslant \operatorname{dim} X$, то имеет место неравенство A-def $X \geqslant 0$. $\mathrm{C}$ другой стороны, если для компакта $X$ для некоторой абелевой группы $G$ имеет место равенство $\operatorname{dim}_{G} X=0$, то компакт $X$ нульмерен, т.е. $\operatorname{dim} X=0$. Это означает, что для всякого компакта $X$ положительной размерности справедлива оценка сверxy: A-def $X \leqslant \operatorname{dim} X-1$. Согласно Дранишникову [48] для любого $n \geqslant 1$ сушествует $n$-мерньй компакт $Y_{n}$, для которого A-def $Y_{n}=n-1$.

Аналогично, из логарифмического неравенства $\operatorname{dim} X \times Y \leqslant \operatorname{dim} X+\operatorname{dim} Y$ вытекает неравенство G-def $X \geqslant 0$. С другой стороны, по теореме Гуревича [49] для всякого конечномерного компакта $X \mathrm{c} \operatorname{dim} X \geqslant 1$ и всякого конечномерного компакта $Y$ справедливо неравенство $\operatorname{dim} X \times Y \geqslant 1+\operatorname{dim} Y$. Это означает, что для всякого компакта $X$ положительной размерности справедлива оценка сверху: G-def $X \leqslant \operatorname{dim} X-1$. На самом деле, одинаковость ограничений на дефекты есть следствие их равенства.

Ю. Кодама [50], В.И. Кузьминов [28] и окончательно А. Н. Дранишников [48] построили пробныле пространства. Именно, в работе А. Н. Дранишникова, Д. Реповша и Е.В. Шепина [39] показано, что построенные Дранишниковьм по групе $G$ и числу $n$ $n$-мерные компакты $Y_{n, G}$ обладают тем свойством, что

$$
\operatorname{dim}_{G} X=\operatorname{dim} X \times Y_{n, G}-\operatorname{dim} Y_{n, G}
$$


для всех компактов $X$ с A-def $X \leqslant n-1$.

Из существования пробных компактов и вычислений в алгебре Бокштейна [39] легко вытекает

Tеорема 5. Для всякого (конечномерного) компакта $X$ справедливо равенство

$$
\text { A-def } X=\text { G-def } X \text {. }
$$

ДокАЗАтельство. Докажем сначала неравенство A-def $X \leqslant \mathrm{G}-\operatorname{def} X$. Для группы $G$ и числа $n=\operatorname{dim} X$ (или даже $n=\mathrm{A}-\operatorname{def} X+1$ ) возьмем пробный компакт $Y_{n, G}$. Тогда

$$
\begin{aligned}
\operatorname{dim} X-\operatorname{dim}_{G} X & =\operatorname{dim} X-\left(\operatorname{dim} X \times Y_{n, G}-\operatorname{dim} Y_{n, G}\right) \\
& =\operatorname{dim} X+\operatorname{dim} Y_{n, G}-\operatorname{dim} X \times Y_{n, G} \leqslant \text { G-def } X .
\end{aligned}
$$

Докажем теперь обратное неравенство A-def $X \geqslant \mathrm{G}$-def $X$. Для доказательства нам потребуется неравенство из работы [39, лемма 2.1, неравенства произведения]: $\min _{G} \operatorname{dim}_{G} X+\operatorname{dim} Y \leqslant \operatorname{dim} X \times Y$. Тогда для произвольного конечномерного компакта $Y$ имеет место цепочка неравенств

$$
\begin{aligned}
& \text { A-def } X=\operatorname{dim} X-\min _{G} \operatorname{dim}_{G} X \geqslant \operatorname{dim} X+\operatorname{dim} Y-\operatorname{dim} X \times Y, \text { поэтому } \\
& \text { A-def } X \geqslant \max _{Y}\{\operatorname{dim} X+\operatorname{dim} Y-\operatorname{dim} X \times Y\}=\text { G-def } X .
\end{aligned}
$$

\section{Формула Бокштейна}

$$
\operatorname{dim}_{G} X=\max \left\{\operatorname{dim}_{H} X: H \in \sigma(G) \subseteq \sigma-\text { базис Бокштейна (основные групшы) }\right\} \text {, }
$$

сводящая вычисление когомологической размерности пространства $X$ по групе $G$ к вычислению когомологических размерностей пространства $X$ по основным групам, показывает, что алгебраический дефект $\mathrm{A}-\operatorname{def} X$ реализуется на одной из основных групп. Неравенства Бокштейна показывают, что алгебраический дефект A-def $X$ peализуется на одной из груп $\mathbb{Z}_{p}$ или $\mathbb{Q}$.

Если в предыдушем доказательстве вместо леммы 2.1 воспользоваться леммой 2.3 из той же работы [39], т.е., в частности, заменить произвольный компакт $Y$ на "геометрически" эквивалентный компакт, являющийся компактификацией Александрова объединения соответствуюших пробных пространств, то можно увидеть, что геометрический дефект G-def $X$ реализуется на одном из $n$-мерных пробных пространств для одной из основных групп $\sigma$ и более того, для одной из групп $\mathbb{Z}_{p} \infty$ или $\mathbb{Q}$.

Tеорема 6. Пусть $f: X \rightarrow Y$ это нульмерное отображение конечномерного компакта $X$ на конечномерный компакт $Y$, причем для группы $G$ справедливо неравенство $\operatorname{dim}_{G} Y \geqslant \operatorname{dim}_{G} X+q$. Тогда для любого $k=1, \ldots, q$ в множестве $B_{k+1}(f)$ имеется такой компакт $Y_{k+1}$, что

$$
\operatorname{dim}_{G} Y_{k+1} \geqslant \operatorname{dim}_{G} Y-k \text {. }
$$


ДокАЗАТЕЛЬСТво. Для числа $n=\operatorname{dim} Y$ и групшы $G$ рассмотрим пробньй компакт $Y_{n, G}$. Отображение $f \times \mathrm{id}: X \times Y_{n, G} \rightarrow Y \times Y_{n, G}$ нульмерно и

$\operatorname{dim} Y \times Y_{n, G}=\operatorname{dim}_{G} Y+\operatorname{dim} Y_{n, G} \geqslant \operatorname{dim}_{G} X+q+\operatorname{dim} Y_{n, G}=\operatorname{dim} X \times Y_{n, G}+q$.

Следовательно, по теореме Фрейденталя-Вайнштейна $[24, \mathrm{Ch} .4, \S 3]$ для любого $k=1, \ldots, q$ в множестве $B_{k+1}(f \times$ id $)$ имеется такой компакт $Z_{k+1}$, что $\operatorname{dim} Z_{k+1} \geqslant$ $\operatorname{dim} Y \times Y_{n, G}-k$. Так как $B_{k+1}(f \times \mathrm{id})=B_{k+1}(f) \times Y_{n, G}$ и проекция на первьй сомножитель является совершенным отображением, то существует такой компакт $Y_{k+1} \subseteq B_{k+1}(f)$, что $Z_{k+1} \subseteq Y_{k+1} \times Y_{n, G}$. Из последнего включения мы и получаем искомое неравенство

$$
\begin{aligned}
\operatorname{dim}_{G} Y_{k+1} & =\operatorname{dim} Y_{k+1} \times Y_{n, G}-\operatorname{dim} Y_{n, G} \geqslant \operatorname{dim} Z_{k+1}-\operatorname{dim} Y_{n, G} \\
& \geqslant \operatorname{dim} Y \times Y_{n, G}-k-\operatorname{dim} Y_{n, G}=\operatorname{dim}_{G} Y-k .
\end{aligned}
$$

ЗАмечАниЕ 8. Доказательство когомологического варианта теоремы Фрейденталя-Вайнштейна мы провели редукцией к соответствующему топологическому утверждению (ведь наши приложения относятся к метрическим компактным пространствам). Однако повторение именно схемы доказательства теоремы Фрейденталя-Вайнштейна позволяет получить более обшие результаты. В связи с совпадением алгебраического и геометрического дефекта компакта $X$ умножение компактов $X$ и $Y$ в доказательстве теоремы 6 на произвольный компакт $Z$ (не пробный) не приводит к более сильному иисловому утверждению. Здесь укажем, что глубокий результат по теореме Гуревича для размерности продолжения получили недавно А. Н. Дранишников и В. В. Успенский [51].

Для доказательства того, что аналог теоремы 2 для $m=n+1$ неверен (в [26] указано, что теорема Дранишникова, применяемая в доказательстве теоремы 2 , при $m=n+1$ неверна), нам нужна

ТЕОРема АЛЕКСАНДРовА. Всякий компакт $X \subset \mathbb{R}^{n+1}$ размерности $\operatorname{dim} X \geqslant n$ является размерно полноценныц.

СледСтвИЕ 12. Для всякого размерностно неполноценного $n$-мерного компакта $X$ для любого нульмерного отображения $f: X \rightarrow \mathbb{R}^{n+1}$ и любого $k=$ $1, \ldots, \mathrm{A}-\operatorname{def} X$ справедливо неравенство

$$
\operatorname{dim} B_{k+1}(f) \geqslant n-k \text {. }
$$

ДокАЗАТЕльство. Пусть $f: X \rightarrow \mathbb{R}^{n+1}$ это нульмерное отображение. Тогда по теореме Гуревича $\operatorname{dim} f(X) \geqslant \operatorname{dim} X-\operatorname{dim} f=n$. По теореме Александрова для любой групш $G$ имеет место неравенство $\operatorname{dim}_{G} f(X)=\operatorname{dim} f(X) \geqslant n$. С другой стороны, существует такая группа $G$, что $\operatorname{dim}_{G} X=\operatorname{dim} X-\mathrm{A}$-def $X=n-\mathrm{A}$-def $X$. Для этой группы $G$ справедливо неравенство

$$
\operatorname{dim}_{G} f(X) \geqslant n=\operatorname{dim}_{G} X+\mathrm{A}-\operatorname{def} X .
$$

Следовательно, искомое неравенство является прямым следствием неравенства Александрова $\operatorname{dim} B_{k+1}(f) \geqslant \operatorname{dim}_{G} B_{k+1}(f)$ и неравенства теоремы 6 .

Следствие 12 показьвает, что теорема Гуревича для $m=n+1$ и $k=2$ допускает и следуюшую форму обрашения. 
ТеОРема 7. Для $n$-мерного компакта $X$ и иелого числа $r$ следующие условия әквивалентны.

(1) Имеет место неравенство $r \geqslant n-1$.

(2) Множество $\mathscr{N}_{r, 2}\left(X, \mathbb{R}^{n+1}\right)$ содержит плотное в пространстве $\mathscr{C}\left(X, \mathbb{R}^{n+1}\right)$ мно жество типа $G_{\delta}$.

(3) Множество всех нульмерных отображений из $\mathscr{N}_{r, 2}\left(X, \mathbb{R}^{n+1}\right)$ плотно в пространстве $\mathscr{C}\left(X, \mathbb{R}^{n+1}\right)$.

ДокАЗАТЕЛЬСТВо. Импликация $(1) \Rightarrow(2)$ является ослабленньм вариантом теоремы Гуревича (для $k=2$ ).

Импликация $(2) \Rightarrow(3)$ вытекает из того, что по теореме Гуревича множество $\mathscr{H}\left(X, \mathbb{R}^{n+1}\right)$ всех регулярно ветвящихся отображений пространства $X$ в пространство $\mathbb{R}^{n+1}$ содержит плотное в пространстве $\mathscr{C}\left(X, \mathbb{R}^{n+1}\right)$ множество типа $G_{\delta}$. Тогда по теореме Бэра и множество $\mathscr{N}_{r, 2}\left(X, \mathbb{R}^{n+1}\right) \cap \mathscr{H}\left(X, \mathbb{R}^{n+1}\right)$ обладает сформулированным свойством.

Для доказательства импликации $(3) \Rightarrow(1)$ рассмотрим два случая.

Пусть $\operatorname{dim} X^{2}=2 n$. Тогда в пространстве $X$ существуют такие дизъюнктные подкомпакты $X_{1}$ и $X_{2}$, что $\operatorname{dim} X_{1} \times X_{2}=2 n$. Возьмем отображения $f_{1}: X_{1} \rightarrow \mathbb{R}^{n+1}$ и $f_{2}: X_{2} \rightarrow \mathbb{R}^{n+1}$, о которых говорится в теореме Дранишникова-Реповша-Щепина. Продолжим отображение $f_{1} \cup f_{2}$ с компакта $X_{1} \cup X_{2}$ на все пространство $X$. Для построенного отображения $g$ из включения $B_{2}\left(g_{\varepsilon}\right) \supseteq g_{\varepsilon}\left(X_{1}\right) \cap g_{\varepsilon}\left(X_{2}\right)$ вытекает неравенство $r \geqslant \min _{\varepsilon} \operatorname{dim}\left(g_{\varepsilon}\left(X_{1}\right) \cap g_{\varepsilon}\left(X_{2}\right)\right) \geqslant n-1$.

Пусть $\operatorname{dim} X^{2}=2 n-1$. Тогда компакт $X$ размерностно неполноценен и по следствию 12 (при $k=1 \leqslant \mathrm{~A}$-def $X$ ) для любого нульмерного отображения $f$ имеет место неравенство $\operatorname{dim} B_{2}(f) \geqslant n-1$.

\section{$\S$ 7. Исторические замечания к формуле Урысона}

Так получилось, что честь создания теории размерности разделили два математика - Павел Самуилович Урысон и Карл Менгер. Уже сподвижники и продолжатели Урысона и Менгера пытались по горячим следам написать историю создания теории размерности. В этом плане уместно указать, что П. С. Александров, друг и часто соавтор П. С. Урысона, и Витольд Гуревич, являвшийся последователем К. Менгера, по просьбе Л. Брауэра написали обзоры для журнала Mathematische Annalen [52], [53], которые отражали вклад Урысона и Менгера в теорию размерности и состояние теории на тот момент.

Истории создания П.С. Урысоном теории размерности посвящены предисловия П.С. Александрова к монографии Гуревича-Волмэна [5] и собранию трудов Урысона [1].

Современное отношение к двум создателям теории размерности, видимо, лучше всего иллюстрируют следующие два ярких примера.

1. Р. Энгелькинг и Р. Поль в статье, посвяшенной В. Гуревичу, пишут:

“Определение размерности было дано независимо П.С. Урысоном в 1922 году и К. Менгером в 1923 году. В зародьшевом состоянии оно появилось ранее в статьях А. Пуанкаре, Брауэра и Лебега. Менгер и Урысон построили теорию размерности компактных метрических пространств; Урысон был впереди Менгера в этой задаче, когда он умер в 1924 году в возрасте 26 лет." 
"Индуктивный подход к размерности, основанный на отделении точек от замкнутых множеств, взятьй в этом определении, и подход, предлагаемьй нижеприводимым свойством (5) (свойством покрытий куба), приводят к одному понятию размерности в классе сепарабельных метрических пространств (см. сноски 3 и 4); вне данного класса это уже неверно. Размерность, введенная Менгером и Урысоном, теперь обычно обозначается как ind и назьвается малой индуктивно размерностью." [10, с. XXI, сноска $^{1}$ ]

"Метод Гуревича давал основные свойства ind, установленные Менгером и Урысоном, существенно более простым путем и позволил расширить теорию размерности с компактных на сепарабельные метрические пространства (такое расширение было достигнуто независимо в то же время Л. Тумаркиным)." [10, с. XXII]

“3 Открытие Лебега привело позже Э. Чеха к введению размерности в смысле покрытий (dim в наших обозначениях)." [10, с. XXIII]

"Процесс продолжения основных теорем теории размерности с компактных на сепарабельные метрические пространства, инициированньй Гуревичем использованием нормальных семейств, был продолжен с помошњю теоремы компактификации, позволяюшей, в частности, расширить характеризацию размерности в терминах конечных замкнутых покрытий порядка $\leqslant n+1$, краеугольный камень теории размерности ${ }^{4}$.

${ }^{4}$ Прозаически эта характеризация означает, что в области сепарабельных метрических пространств малая индуктивная размерность и размерность в смысле покрытий совпадают." [10, с. XXIII]

Обратим внимание на два момента.

а. Приведенные отрьвки невольно заставляют читателя приписать основной вклад Урысона в теорию размерности (см. введение) другим замечательным математикамвведение размерности $\operatorname{dim} \ni$. Чеху, а тождество $\operatorname{dim} X=\operatorname{ind} X=\operatorname{Ind} X$ В. Гуревичу.

б. При изложении фактов Урысон признается идушим впереди и упоминается первым. При общем же обсуждении истории создания теории размерности происходит "инверсия".

Впрочем, в статье, посвяшенной В. Гуревичу, являвшемуся последователем дела Менгера, такая "инверсия" еще понятна. Приведем пример "расширения", история возникновения которого автору совершенно непонятна и в которой, видимо, разобраться уже невозможно.

2. Перечисляя заслуги Урысона, и только Урысона, П.С. Александров пишет $[5$, с. 10$],[1$, т. 1, с. 33$]$ :

"Среди теорем, доказанных Урысоном и не доказанных Менгером, отметим следуюшие:

2) В главе 6 Урысон доказьвает свою формулу

$$
\operatorname{ind}(A \cup B) \leqslant \operatorname{ind} A+\operatorname{ind} B+1
$$

и вьводит из нее теорему о том, что $n$-мерное множество может быть представлено как сумма $n+1$ нульмерного множества и не может быть представлено как сумма меньшего числа нульмерных множеств. При этом даются очень точные оценки класса слагаемых с точки зрения дескриптивной классификации. Этой теоремы, одной из основных в теории размерности, у Менгера тоже нет." 
К сожалению, местоимение “этой” в применении к трем теоремам (особенно в свете нижеследующего) оставляет место неясностям.

а. Автор данных строк в ранних работах К. Менгера не смог найти приведенную формулу.

б. В. Гуревич в статье, дающей конспект менгеровской теории размерности, пишет $[53$, c. $72(135)]$ :

"Из теоремы суммы легко получается теорема Урысона о том, что всякое $n$-мерное $\sigma$-компактное пространство является суммой $n+1$, но не меньше, нульмерного множества."

В. Гуревич дает идейно очень ясное, но технически очень неполное доказательство сформулированной теоремы. Так, в доказательстве невозможности представления в виде меньшего числа слагаемых, т.е. фактически в доказательстве формулы $(y)$, вообще не возникают ограничения на область ее справедливости.

Но в данном случае нас интересует только тот факт, что, излагая теорию Менгера, В. Гуревич называет соответствующий факт теоремой Урысона.

Впрочем, и в своей оригинальной работе по продолжению теории размерности с компактных на сепарабельные метрические пространства с помошью понятия нормального семейства, упоминавшейся вьше при цитировании Р. Энгелькинга и Р. Поля, В. Гуревич пишет $\left[54\right.$, с. $761(82)$, сноска $\left.^{27}\right]$ :

"Относительно компактных замкнутых множеств эта теорема была объявлена Урысоном без доказательства [55]. Доказательство Урысона, опубликованное в Fund. Math. [15], во время печатания этой работы автору не было известно."

в. Отличающийся исключительной точностью, Ричард Энгелькинг в своей монографии по теории размерности связьвает обсуждаемую формулу только с именем Урысона.

Казалось бы, что в таком конкретном случае вопроса с атрибутикой имен создателей теории размерности не должно быть. Но процитируем теперь монографию П. С. Александрова и Б. А. Пасьнкова.

“3. ФормУлА УРысонА-МенГеРА. Самым замечательным фактом, касающимся малой индуктивной размерности, является, бесспорно, формула Урысона-Менгера $(y)$ для любых множеств $A$ и $B$ в произвольном наследственно нормальном пространстве $X . "[9$, с. 180$]$

"Одним из основных результатов в теории размерности наследственно нормальных пространств является формула Урысона-Менгера

$$
\operatorname{dim}(A \cup B) \leqslant \operatorname{dim} A+\operatorname{dim} B+1,
$$

доказанная Смирновым [56] для любых множеств $A, B$ наследственно нормального пространства $X . "[9$, с. 397]

"В случае произвольных нормальных пространств неравенство Урысона-Менгера установлено Зарелуа [57]." [9, с. 401, сноска*]

Отметим, что как Ю. М. Смирнов, так и А. В. Зарелуа пишут в своих работах о неравенстве Урысона, а не о неравенстве Урысона-Менгера. Смена терминологии произошла после опубликования монографии Александрова-Пасьнкова.

Как сказал автору данных строк Б. А. Пасынков, весь материал, относящийся к наследственно нормальным пространствам, из которого взяты приведенные вьше цита- 
ты, написан лично П. С. Александровьм. Понять причину, по которой П. С. Александров изменил свое мнение (а может, описался?) в вопросе, который, судя по всему, был ему далеко не безразличен, представляется сегодня почти невозможным. Во всяком случае, приведенные выше выдержки из работ Александрова, а также приводимьй ниже комментарий Александрова к мемуару Урысона с несомненностью показьвают, что сам Александров придавал указанным результатам большое значение и, как показывает последующее развитие теории размерности, в этом он был, несомненно, прав.

"Едва ли можно согласиться с автором (П. С. Урысоном - прим. автора), что, например, теорема о том, что всякое $n$-мерное множество может быть представлено как сумма $n+1$ (но не меньшего числа) множеств размерности нуль, есть теорема 'второстепенного значения'." [1, с. 491]

В статье Б.П. Двалишвили [58], написанной после опубликования монографии Александрова-Пасьнкова, в категории битопологических пространств получена уже “формула Урысона-Менгера”. Л.Г. Замбахидзе [59] ввел функции размерностного типа I и r, указьвающие степень близости данной топологии к бикомпактной (периферически бикомпактной) топологии, и доказал для инвариантов I и r уже “формулу Менгера-Урысона".

Совершенно особое положение формула Урысона (но уже как “формула Урысона-Менгера" или даже “формула Менгера-Урысона") приобрела в рамках теории размерности благодаря рождению новой дисциплины - “теории продолжения" в терминологии А.Н. Дранишникова [60] и “теории экстмерности” в терминологии Е.В. Шепина [30]. Здесь очень важно подчеркнуть, что әкстмерные результаты позволили получить новые неожиданные соотношения в рамках классической теории размерности. Работа А.Н. Дранишникова [26] дает удивительный пример "цементирования" теорией экстмерности результатов по существованию отображений общего положения, формуле (log) и формуле Урысона-Менгера $(У)$.

Как А. Н. Дранишников, Д. Реповш и Е. В. Шепин [61], так и Ю. Дыдак [62] отмечают, что они решают проблему 10 из обзора В. И. Кузьминова [28]. Однако В. И. Кузьминов спрашивает о справедливости когомологической формулы Урысона. В работах же [26], [61]-[64] говорится о формуле (неравенстве, теореме) Урысона-Менгера и даже чаще - Менгера-Урысона.

В заключение подчеркнем, что именно воспитаники школы Александрова (школы Урысона-Александрова, по мнению самого Александрова - "Положение П. С. Урысона в истории математической науки определяется прежде всего тем, что он явился создателем советской топологии" $[1$, т. 1, с. 20]), читавшие (и, может быть, неоднократно) предисловие Александрова к монографии Гуревича-Волмэна, но еще более внимательно читавшие монографию Александрова-Пасынкова, ввели в устойчивьй математический оборот вместо термина "неравенство Урысона" термин "неравенство Урысона-Менгера".

Приведенная история является уникальным примером иронии судьбы, когда именно благодаря математику, подчеркивавшему обшепринятое мнение, что другой математик не имеет никакого отношения к одному конкретному результату, этот результат и стал впоследствии ассоциироваться еще и с именем второго математика.

Автору приятно выразить свою благодарность А. Ю. Воловикову, А. Н. Дранишникову, Б.А. Пасынкову, В. В. Федорчуку и Е. В. Щепину за помош. Особая благодарность Х. Цишангу за приглашение в мае-июле 1995 г. в Ruhr-Universität Bochum, где 
началась эта работа, и Х. Штайнлайну из Ludwig-Maximilians-Universität München, в результате доклада на семинаре которого в июле 1995 года автор осознал универсальность метода обшего положения.

\section{СПИСОК ЛИТЕРАТУРЫ}

[1] Урысон П.С. Труды по топологии и другим областям математики. Т. 1 и 2. М.: Гостехиздат, 1951.

[2] Красносельский М. А. Топологические методы в теории нелинейных интегральных уравнений. М.: Гостехиздат, 1956.

[3] Sangwine-Yager J. R. Mixed volumes // Handbook of Convex Geometry / ed. P. M. Gruber, J. M. Wills. Amsterdam: Elsevier Sci. Publ., 1993. P. 43-71.

[4] Тихомиров В. М. Некоторые вопросы теории приближений. М.: Изд-во МГУ, 1976.

[5] Гуревич В., Волмэн Г. Теория размерности. М.: ИЛ, 1948.

[6] Lebesgue H. Sur la non applicabilité de deux domaines appartenant respectivement à deux espaces, de $n$ et $n+p$ dimensions // Math. Ann. 1911. V. 70. P. 166-168.

[7] Lebesgue H. Sur les correspondances entre les points de deux espaces // Fund. Math. 1921. V. 2. P. 256-285.

[8] Brouwer L. E. J. Über den natürlichen Dimensionsbegriff // J. Reine Angew. Math. 1913. V. 142. P. $146-152$.

[9] Александров П.С., Пасынков Б.А. Введение в теорию размерности. М.: Наука, 1973.

[10] Engelking R., Pol R. Hurewicz's contributions to dimension theory // Collect. Works of Witold Hurewicz / ed. K. Kuperberg. Providence, RI: Amer. Math. Soc., 1995. P. XXI-XXVIII.

[11] Pontrjagin L. Sur une hypothèse fondamentale de la dimension // C. R. Acad. Sci. 1930. V. 190. P. 1105-1107.

[12] Болтянский В. Г. О теореме сложения размерностей // УМН. 1951. Т. 6. № 3. С. 99-129.

[13] Бокштейн М. Ф. Гомологические инварианты топологических пространств. I. II. // Труды МMO. 1956. Т. 5. С. 3-80; 1957. Т. 6. С. 3-133.

[14] Бабенко К.И. Основы численного анализа. М.: Наука, 1986.

[15] Urysohn P. Mémoire sur les multiplicités cantoriennes // Fund. Math. 1925. V. 7. P. $30-137$; 1926. V. 8. P. 225-359.

[16] Alexandroff P. Über die Urysonsche Konstanten // Fund. Math. 1933. V. 20. P. 140-150.

[17] Богатый С. А. Теорема Люстерника-Шнирельмана и $\beta f / /$ Фундам. и прикл. матем. 1998. T. 4. № 1. C. 11-38.

[18] Макеев В.В.О некоторых свойствах непрерьвных отображений сфер и задачах комбинаторной геометрии // Геометрические вопросы теории функций и множеств. Калинин: Калинин. гос. ун-т, 1986. С. 75-85.

[19] Бабенко И.К., Богатый С.А. К отображению сферы в евклидово пространство // Матем. заметки. 1989. Т. 46. № 3. С. 3-8.

[20] Воловиков А. Ю. Теорема типа Буржена-Янга для $\mathbb{Z}_{p}^{n}$-действия // Матем. сб. 1992. T. 183. № 7. C. $115-144$.

[21] Bogatyi S. A. The Knaster problem, the Borsuk theorem and cyclic systems // Topol. and Appl. Intern. Topol. Conf. dedic. P.S. Alexandroff's 100th Birthday. Moscow, May 27-31, 1996. Moscow: PHASIS. P. 167-169.

[22] Hurewicz W. Dimensionstheorie und Cartesische Räume // Koninklijke Akademie van Wetenschappen. Proceedings of the Section of Sciences 1931. V. 34. P. 399-400; перепечатано в Collect. Works of Witold Hurewicz. Providence, RI: Amer. Math. Soc. 1995. P. 253-255.

[23] Hurewicz W. Über Abbildungen von endlichdimensionalen Räumen auf Teilmengen Cartesischer Räume // Sitzungsberichte der Preußischen Akademie der Wissenschaften 1933. V. 4. P. 754-768; перепечатано в Collect. Works of Witold Hurewicz. Providence, RI: Amer. Math. Soc. 1995. P. 295-309.

[24] Engelking R. Dimension Theory. Amsterdam: North-Holland, 1978. 
[25] Dranišnikov A.N., Repovš D., Ščepin E. V. On intersections of compacta of complementary dimensions in Euclidean space // Topol. Appl. 1991. V. 38. P. 237-253.

[26] Dranishnikov A. N. On the dimension of the product of two compacta and the dimension of their intersection in general position in Euclidean space // Preprint.

[27] Nöbeling. Abstract on the article [21] // Zentralblatt für Math. und ihre Grenzgeb. 1931. V. 1. P. 409; перепечатано в Collect. Works of Witold Hurewicz. Providence, RI: Amer. Math. Soc. 1995. P. 255.

[28] Кузьминов В.И. Гомологическая теория размерности // УМН. 1968. Т. 23. №5. C. 3-49.

[29] Dranishnikov A. N., West J. On compacta that intersect unstably in Euclidean space // Topol. Appl. 1992. V. 43. P. 181-187.

[30] Шепин Е. В. Арифметика теории размерности // УМН. 1998. Т. 53. № 5.

[31] Dranishnikov A. N., Repovš D., Ščepin E. V. Transversal intersection formula for compacta // Preprint.

[32] Tverberg H. A generalization of Radon's theorem // J. London Math. Soc. 1966. V. 41. P. $123-128$.

[33] Van Kampen E. R. Komplexe in euklidischen Raumen // Abh. Math. Sem. Hamburg. 1932. V. 9. P. 72-78.

[34] Flores A. Über $n$-dimensionale Komplexe die im $R_{2 n+1}$ absolut selbstverschlungen sind // Ergegb. Math. Kolloq. 1933/34. V. 6. P. 4-7.

[35] Sarkaria K.S. A generalized van Kampen-Flores theorem // Proc. Amer. Math. Soc. 1991. V. 111. № 2. P. 559-565.

[36] Воловиков А. Ю. К теореме ван Кампена-Флореса // Матем. заметки. 1996. Т. 59. № 5. C. 663-670.

[37] Cohen F., Lusk E. L. Configuration-like spaces and the Borsuk-Ulam theorem // Proc. Amer. Math. Soc. 1976. V. 56. P. 313-317.

[38] Болтянский В.Г. О размерной полноценности компактов // Докл. АН СССР. 1949. T. 67. № 5. C. $775-777$.

[39] Dranišnikov A. N., Repovš D., Ščepin E.V. On approximation and embedding problems for cohomological dimension // Topol. Appl. 1994. V. 55. № 1. P. 67-86.

[40] Пасынков Б. А. Об универсальных бикомпактных и метрических пространствах данной размерности // Fund. Math. 1967. V. 60. Р. 285-308.

[41] Summerhill R. R. General position for closed subsets of Euclidean space // Gen. Topol. Appl. 1973. V. 3. P. 339-345.

[42] Radon J. Mengen konvexer Körper, die einen gemeinsamen Punkt enthalten // Math. Ann. 1921. V. 83. P. 113-115.

[43] Bajmóczy E. G., Bárány I. On common generalization of Borsuk's and Radon's theorem // Acta Math. Acad. Sci. Hungar. 1979. V. 34. P. 347-350.

[44] Bárány I., Shlossman S. B., Szücs A. On topological generalization of a theorem of Tverberg // J. London Math. Soc. 1981. V. 23. P. 158-164.

[45] Воловиков А. Ю. К топологическому обощению теоремы Тверберг // Матем. заметки. 1996. T. 59. № 3. С. 454-456.

[46] Борсук К. Замечания к вложимости множеств в евклидовы пространства // Труды III Всесоюзн. матем. съезда. 1959. T. IV. C. 197-198.

[47] Болтянский В. Г. Отображения компактов в эвклидовыпространства // Изв. АН СССР. Сер. матем. 1959. Т. 23. С. 871-892.

[48] Дранишников А.Н. Гомологическая теория размерности // УМН. 1988. Т. 43. № 4. C. $11-55$.

[49] Hurewicz W. Sur la dimension des produits Cartésiens // Ann. of Math. 1935. V. 36. № 1. P. 194-197; перепечатано в Collect. Works of Witold Hurewicz. Providence, RI: Amer. Math. Soc. 1995. P. 335-339.

[50] Kodama Y. On a problem of Alexandroff concerning the dimension of product spaces, II // J. Math. Soc. Japan. 1959. V. 11. P. 94-111. 
[51] Dranishnikov A. N., Uspenskij V.V. Light maps and extensional dimension // Preprint.

[52] Alexandroff P. Darstellung der Grundzüge der Urysohnschen Dimensionstheorie // Math. Ann. 1928. V. 98. №1. P. 31-63.

[53] Hurewicz W. Grundriß der Mengerschen Dimensionstheorie // Math. Ann. 1928. V. 98. № 1. P. 64-88; перепечатано в Collect. Works of Witold Hurewicz. Providence, RI: Amer. Math. Soc. 1995. P. 127-151.

[54] Hurewicz W. Normalbereiche und Dimensionstheorie // Math. Ann. 1927. V. 96. P. 736-764; перепечатано в Collect. Works of Witold Hurewicz. Providence, RI: Amer. Math. Soc. 1995. P. 57-86.

[55] Urysohn P. Les multiplicités cantoriennes // C. R. Acad. Sci. 1922. V. 175. P. 440-442.

[56] Смирнов Ю.М. Некоторые соотношения в теории размерности // Матем. сб. 1951. T. 29. № 1. C. 157-172.

[57] Зарелуа А. В. О равенстве размерностей // Матем. сб. 1963. Т. 62. № 3. С. 295-319.

[58] Двалишвили Б. П. О размерности и некоторых других вопросах теории битопологических пространств // Тр. Тбилис. матем. ин-та. 1977. Т. 56. С. 15-51.

[59] Замбахидзе Л. Г. О функциях размерностного типа // Тр. Тбилис. матем. ин-та. 1977. T. 56. C. $52-98$.

[60] Dranishnikov A. N. On intersection of compacta in Euclidean space. II // Proc. Amer. Math. Soc. 1991. V. 113. № 4. P. 1149-1154.

[61] Dranishnikov A. N., Repovš D., Ščepin E. On the failure of the Urysohn-Menger sum formula for cohomological dimension // Proc. Amer. Math. Soc. 1994. V. 120. P. $1267-1270$.

[62] Dydak J. Cohomological dimension and metrizable spaces. II // Trans. Amer. Math. Soc. 1996. V. 348. № 4. P. 1647-1661.

[63] Dranishnikov A., Repovš D. The Urysohn-Menger sum formula: An extension of the Dydak-Walsh theorem to dimension one // J. Austral. Math. Soc. Ser. A. 1995. V. 59. № 2. P. 273-282.

[64] Dydak J. Union theorem for cohomological dimension: A simple counterexample // Proc. Amer. Math. Soc. 1994. V. 121. № 1. P. 295-297.

Московский государственный

Поступила в редакцию университет им. М. В. Ломоносова

28.04 .1998

E-mail: bogatyi@nw.math.msu.su 